\title{
Econometric analysis of realised covariation: high frequency covariance, regression and correlation in financial economics
}

\author{
Ole E. BARndorfF-Nielsen \\ The Centre for Mathematical Physics and Stochastics (MaPhySto), \\ University of Aarhus, Ny Munkegade, DK-8000 Aarhus C, Denmark \\ oebn@mi . aau.dk \\ NeIL SHEPHARD \\ Nuffield College, Oxford OX1 1NF, UK \\ neil.shephard@nuf .ox.ac.uk
}

First draft: November 2001

This draft: 18th March 2002

www . LevyProcess . org

\begin{abstract}
This paper analyses multivariate high frequency financial data using realised covariation. We provide a new asymptotic distribution theory for standard methods such as regression, correlation analysis and covariance. It will be based on a fixed interval of time (e.g. a day or week), allowing the number of high frequency returns during this period to go to infinity. Our analysis allows us to study how high frequency correlations, regressions and covariances change through time. In particular we provide confidence intervals for each of these quantities.
\end{abstract}

Keywords: Power variation; Realised correlation; Realised covolatility; Realised regression; Realised variance; Semimartingales; Covolatility.

\section{Introduction}

\subsection{Motivation, definitions and assumptions}

This paper provides a new econometric analysis of the covariance between asset returns. Based on a fixed interval of time (e.g. a trading day or a calendar month), our asymptotic theory allows the number of high frequency returns during this period to go to infinity. This new theory allows us to study how covariances, correlations and regression coefficients change through time by computing these quantities over non-overlapping intervals of time and providing confidence limits for them.

The new econometrics is motivated by the advent of complete records of quotes or transaction prices for many financial assets. Although market microstructure effects (e.g. discreteness of prices, bid/ask bounce, irregular trading etc.) means that there is a mismatch between asset 
pricing theory based on semimartingales and the data at very fine time intervals (see, for example, Bai, Russell, and Tiao (2000)) it does suggest the desirability of establishing an asymptotic distribution theory for estimators as we use more and more highly frequent observations.

Here we suppose there are $M$ intra- $\hbar$ observations during each $\hbar>0$ time period and that log-price of a $q$ dimensional vector of assets is written as $y^{*}$. The high frequency observations will be defined as

$$
y_{j, i}=y^{*}\left((i-1) \hbar+\hbar j M^{-1}\right)-y^{*}\left((i-1) \hbar+\hbar(j-1) M^{-1}\right),
$$

the $j$-th intra- $\hbar$ return for the $i$-th period (e.g. if $\hbar$ is a day, $M=1330$, then this is the return for the $j$-th minute on the $i$-th day). We will often write the $k$-th element of the vector $y_{j, i}$ as $y_{j, i(k)}$. Then our focus of attention will be on

$$
\left[y_{M}^{*}\right]_{i}=\sum_{j=1}^{M} y_{j, i} y_{j, i}^{\prime}=\left\{\sum_{j=1}^{M} y_{j, i(k)} y_{j, i(l)}\right\}_{k, l=1, \ldots, q} .
$$

In econometrics $\left[y_{M}^{*}\right]_{i}$ is labelled the realised covariation matrix. In this paper we will establish the asymptotic distribution of $\left[y_{M}^{*}\right]_{i}$ as $M \rightarrow \infty$, so providing a guide to its behaviour for a finite value of $M$. The result is important in its own right of course, but it also implies a distribution theory for quantities derived from the realised covariation matrix. Examples of this include realised regression, realised correlation and more sophisticated quantities, including estimated optimal portfolio weights and the estimated efficiency frontier for mean-variance investors.

Under very weak assumptions the probability limit of $\left[y_{M}^{*}\right]_{i}$ has been known for many years using the theory of quadratic covariation (e.g. Jacod and Shiryaev (1987, p. 55), Back (1991), Andersen, Bollerslev, Diebold, and Labys (2001a), Meddahi (2002)). Here we remind readers of the substance of that theory, before we go beyond this to develop the asymptotic distribution theory.

A $q$ dimensional special semimartingale $y^{*}$ can be uniquely decomposed as

$$
y^{*}(t)=\alpha^{*}(t)+m^{*}(t),
$$

where $\alpha^{*}(t)$, a drift term, is a predictable process with locally bounded variation paths and $m^{*}(t)$ is a local martingale. For an excellent discussion of probabilistic aspects of this see Protter (1990). Back (1991) discusses why constraining ourselves to live within the class of special semimartingales makes sense from an economic viewpoint.

One of the most important aspects of semimartingales is the quadratic covariation (QV) matrix defined as

$$
\left[y^{*}\right](t)=\mathrm{p}-\lim _{M \rightarrow \infty} \sum_{j=0}^{M-1}\left\{y^{*}\left(t_{j+1}\right)-y^{*}\left(t_{j}\right)\right\}\left\{y^{*}\left(t_{j+1}\right)-y^{*}\left(t_{j}\right)\right\}^{\prime},
$$


for any sequence of partitions $t_{0}=0<t_{1}<\ldots<t_{M}=t$ with $\sup _{j}\left\{t_{j+1}-t_{j}\right\} \rightarrow 0$ for $M \rightarrow \infty$. Here $\mathrm{p}-$ lim denotes the probability limit of the sum. Later it will be helpful to label the $k, l$-th element of the $\mathrm{QV}\left[y^{*}\right](t)$ as the quadratic covariance

$$
\left[y_{(k)}^{*}, y_{(l)}^{*}\right](t)=\mathrm{p}-\lim _{M \rightarrow \infty} \sum_{j=0}^{M-1}\left\{y_{k}^{*}\left(t_{j+1}\right)-y_{k}^{*}\left(t_{j}\right)\right\}\left\{y_{l}^{*}\left(t_{j+1}\right)-y_{l}^{*}\left(t_{j}\right)\right\} .
$$

The definition of QV immediately implies

$$
\left[y_{M}^{*}\right]_{i} \stackrel{p}{\rightarrow}\left[y^{*}\right](\hbar i)-\left[y^{*}\right](\hbar(i-1)), \quad \text { as } M \rightarrow \infty
$$

meaning realised covariation consistently estimates increments of QV.

In general (e.g. Jacod and Shiryaev (1987, p. 55))

$$
\left[y^{*}\right](t)=\left[y^{* c}\right](t)+\sum_{0 \leq s \leq t} \Delta y^{*}(s) \Delta y^{*}(s)^{\prime}
$$

where $y^{* c}$ is the continuous component of $y^{*}$ and $\Delta y^{*}(t)=y^{*}(t)-y^{*}(t-)$ are the jumps at time $t$. In the context of the semimartingale (3) the QV becomes

$$
\begin{aligned}
{\left[y^{*}\right](t)=} & {\left[m^{*}\right](t)+\sum_{0 \leq s \leq t} \Delta \alpha^{*}(s) \Delta \alpha^{*}(s)^{\prime}+\sum_{0 \leq s \leq t} \Delta m^{*}(s) \Delta \alpha^{*}(s)^{\prime} } \\
& +\sum_{0 \leq s \leq t} \Delta \alpha^{*}(s) \Delta m^{*}(s)^{\prime}
\end{aligned}
$$

the QV of $m^{*}$ plus terms which are influenced by the jumps in $\alpha^{*}$ and $m^{*}$. If $\alpha^{*}$ is continuous then we obtain the simplification

$$
\left[y^{*}\right](t)=\left[m^{*}\right](t)
$$

which holds irrespective of the presence of jumps in the local martingale component ${ }^{1}$.

The result (10) is powerful for it does not depend upon the model for $m^{*}$ or $\alpha^{*}$, only on the assumption that $\alpha^{*}$ is continuous. In the univariate case it is discussed in the econometric literature by independent and concurrent work by Comte and Renault (1998), Barndorff-Nielsen and Shephard (2001a) and Andersen and Bollerslev (1998). It was later developed and applied in some empirical work by Andersen, Bollerslev, Diebold, and Labys (2001b). See also BarndorffNielsen and Shephard (2001a) and Andersen, Bollerslev, Diebold, and Labys (2001a) for a discussion of the multivariate case and Andersen, Bollerslev, and Diebold (2002) for an incisive survey of this area. Andersen, Bollerslev, Diebold, and Ebens (2001) discusses the use of the multivariate theory in the context of equity prices.

The above theoretical framework is too general for us to be able to derive a distribution theory for it. As a result we have had to specialise. In particular we make three assumptions.

\footnotetext{
${ }^{1}$ This holds as the quadratic variation of any continuous, locally bounded variation process is zero.
} 
1. That $m^{*}$ is a multivariate stochastic volatility $(\mathrm{SV})$ process

$$
m^{*}(t)=\int_{0}^{t} \Sigma^{1 / 2}(u) \mathrm{d} w(u),
$$

where $\Sigma^{1 / 2}(t)$, is the instantaneous or spot covolatility matrix, and $w$ is standard multivariate Brownian motion ${ }^{2}$. We call $\Sigma$ the spot covariance matrix process and assume $\Sigma(t)$ is positive semi-definite for all values of $t$ and that each element of $\Sigma$ is a local bounded variation process. Whatever the model for $\Sigma$ the implied $m^{*}$ must be a continuous local martingale.

2. For every $\nu=1, \ldots, q$ the mean process $\alpha_{\nu}^{*}$ is càglàd and satisfies (pathwise)

$$
\delta^{-3 / 4} \max _{1 \leq j \leq M}\left|\alpha_{\nu}^{*}(j \delta)-\alpha_{\nu}^{*}((j-1) \delta)\right|=o(1),
$$

in $\delta$. This condition implies that the $\alpha^{*}$ process is continuous and so is predictable. In the results which follow, we refer to (12) as condition (A).

3. The joint $\alpha^{*}, \Sigma$ process is independent of $w$.

Conditions 1 and 2 imply this model structure is a continuous, special semimartingale with

$$
y^{*}(t)=\alpha^{*}(t)+\int_{0}^{t} \Sigma^{1 / 2}(u) \mathrm{d} w(u) .
$$

Condition 3 is a strong, undesirable additional assumption for it rules out empirically important dynamic effects such as leverage (e.g. Black (1976) and Nelson (1991)). We will comment in detail about the import of this assumption in Section 4.3 of this paper.

A simple, but important, example of a general $\alpha^{*}$ process which satisfies $(\mathbf{A})$ is where $\alpha^{*}(t)=\mu t+\Sigma^{*}(t) \beta$, where

$$
\Sigma^{*}(t)=\int_{0}^{t} \Sigma(u) \mathrm{d} u .
$$

This links the mean process to the covariance. It has the feature that for all possible models for $\Sigma, \partial \alpha^{*}(t) / \partial t=\mu+\Sigma(t) \beta$. Differentiability of $\alpha^{*}$ is a much stronger assumption than $(\mathbf{A})$.

Importantly, for this model class $\left[y^{*}\right](t)=\Sigma^{*}(t)$, due to condition $(\mathbf{A})$ ruling out the possibility of jumps in $\alpha^{*}$ and (11). This implies $\left[y_{M}^{*}\right]_{i} \stackrel{p}{\rightarrow} \Sigma_{i}$, where $\Sigma_{i}=\Sigma^{*}(i \hbar)-\Sigma^{*}\{(i-1) \hbar\}$. In econometrics $\Sigma^{*}(t)$ is called integrated covariance matrix, while we call $\Sigma_{i}$ actual covariance matrix (Andersen, Bollerslev, and Diebold (2002) call this the notional covariance matrix). Both quantities play a central role in the probabilistic analysis of SV models. Reviews of the literature on this topic are given in Shephard (1996) and Ghysels, Harvey, and Renault (1996).

\footnotetext{
${ }^{2}$ The assumption of a continuous time SV model looks, at first sight, contrived. However, in the univariate case all local martingales with continuous sample paths can be written as time changed Brownian motions (e.g. Protter (1990, p. 81)). If the time change is itself differentiable, then this produces a univariate SV model. To our knowledge the multivariate version of the this result has not, however, been established.
} 
The quadratic variation result implies $\partial\left[y^{*}\right](t) / \partial t$ equals $\Sigma(t)$, which means in this context we can view $\Sigma$ as observable given the paths of $y^{*}$. This implies that $\Sigma(t) \mathrm{d} t$ is the conditional (given the natural filtration) covariance matrix of the infinitesimal return $\mathrm{d} y^{*}(t)$. As a result $\Sigma^{*}(t)$ is the integrated conditional covariance matrix, while $\alpha^{*}(t)$ has the interpretation as the integrated conditional mean process. The economic implications of these issues are discussed at more length in Back (1991) and Andersen, Bollerslev, Diebold, and Labys (2001a).

\subsection{Contribution and literature}

In the special case of $y^{*}$ being univariate, Barndorff-Nielsen and Shephard (2002a) have recently established the following distributional results for the realised variance ${ }^{3}\left[y_{M}^{*}\right]_{i}$, considerably strengthening the consistency result implied by (4). In particular they showed that, under some weak regularity assumptions discussed in the next section, the following three results hold. The first is that

$$
\frac{\sqrt{\frac{M}{\hbar}}\left\{\left[y_{M}^{*}\right]_{i}-\Sigma_{i}\right\}}{\sqrt{2 \int_{(i-1) \hbar}^{i \hbar} \Sigma^{2}(u) \mathrm{d} u}} \stackrel{L}{\rightarrow} N(0,1) .
$$

The second result is that

$$
\frac{\left[y_{M}^{*}\right]_{i}-\Sigma_{i}}{\sqrt{\frac{2}{3} \sum_{j=1}^{M} y_{j, i}^{4}}} \stackrel{L}{\rightarrow} N(0,1) .
$$

These two limit theorems are linked together by the third result which is that

$$
\frac{M}{3 \hbar} \sum_{j=1}^{M} y_{j, i}^{4} \stackrel{p}{\rightarrow} \int_{(i-1) \hbar}^{i \hbar} \Sigma^{2}(u) \mathrm{d} u .
$$

The result (15) is feasible, while (14) is perhaps more informative from a theoretical viewpoint. In particular the two results imply:

1. $\left[y_{M}^{*}\right]_{i}$ converges to $\Sigma_{i}$ at rate $\sqrt{M}$.

2. The limit theorem is unaffected by the form of the drift process.

3. Knowledge of the volatility dynamics is not required in order to use this theory.

4. The fourth moment of returns need not exist for the asymptotic normality to hold.

5. The volatility process $\Sigma(t)$ can be non-stationary, including intra-day effects.

\footnotetext{
${ }^{3}$ Sums of squared returns are often called realised volatility in econometrics, while we use the name realised variance for that term and realised volatility for the corresponding square root. The use of volatility to denote standard deviations rather than variances is standard in financial economics. See, for example, the literature on volatility and variance swaps, which are derivatives written on realised volatility or variance, which includes Demeterfi, Derman, Kamal, and Zou (1999), Howison, Rafailidis, and Rasmussen (2000) and Chriss and Morokoff (1999). We have chosen to follow this nomenclature rather than the one more familiar in econometrics. Confidence intervals for the realised volatility follow by square rooting the confidence intervals for the realised variance.
} 
6. $\left[y_{M}^{*}\right]_{i}-\Sigma_{i}$ has a mixed Gaussian limit implying that it will have heavier tails than a normal.

7. The magnitude of the error $\left[y_{M}^{*}\right]_{i}-\Sigma_{i}$ is likely to be large in times of high volatility.

These results are illustrated on empirical data in Barndorff-Nielsen and Shephard (2002b), while Monte Carlo evidence of the accuracy of these asymptotic approximations, and an asymptotically equivalent feasible alternative

$$
\frac{\log \left[y_{M}^{*}\right]_{i}-\log \Sigma_{i}}{\sqrt{\frac{2}{3}\left(\sum_{j=1}^{M} y_{j, i}^{2}\right)^{-2} \sum_{j=1}^{M} y_{j, i}^{4}}} \stackrel{L}{\rightarrow} N(0,1),
$$

is studied in Barndorff-Nielsen and Shephard (2001b). The conclusion of that paper is to prefer the form (17) which has significantly superior coverage properties compared to (15). See also Meddahi (2002) and Andreou and Ghysels (2001) who have interesting additional insights into these and other results about the accuracy of $\left[y_{M}^{*}\right]_{i}$.

In independently and concurrent work Meddahi (2002) has studied the first two moments of the difference between elements of the multivariate $\left[y_{M}^{*}\right]_{i}$ and $\Sigma_{i}$. This is the only other paper we know of which has discussed the properties of $\left[y_{M}^{*}\right]_{i}-\Sigma_{i}$.

These results are also quite closely related to the work of Foster and Nelson (1996) (note also the work of Genon-Catalot, Laredo, and Picard (1992), Florens-Zmirou (1993) and Hansen (1995)). In the univariate SV case, where the volatility follows a scalar diffusion, they provided an asymptotic distribution theory for an estimator of $\Sigma(t)$, the spot (not integrated) variance. Their idea was to compute a local variance from the lagged data, e.g.,

$$
\widehat{\Sigma}(t)=\hbar^{-1} \sum_{j=1}^{M}\left\{y^{*}\left(t-\hbar j M^{-1}\right)-y^{*}\left(t-\hbar(j-1) M^{-1}\right)\right\}^{2} .
$$

They then studied its behaviour as $M \rightarrow \infty$ and $\hbar \downarrow 0$ under some assumptions. This "double asymptotics" yields a Gaussian limit theory so long as $\hbar \downarrow 0$ and $M \rightarrow \infty$ at the right, related rates. Of course this type of argument is familiar also in nonparametric econometrics (e.g. Pagan and Ullah (1999)). The double asymptotics makes it harder to use in practice than our own simpler analysis, which just needs $M \rightarrow \infty$. This is made possible because our goal is to estimate the easier integrated covariation rather than the harder spot covariation.

In this paper we extend the univariate results in (14), (15) and (16) to cover the multivariate case. The contributions of the paper will be as follows:

- Our main result is that as $M \rightarrow \infty$, conditioning on the path of $\alpha^{*}$ and $\Sigma$,

$$
\sqrt{\frac{M}{\hbar}}\left\{\operatorname{vech}\left(\left[y_{M}^{*}\right]_{i}\right)-\operatorname{vech}\left(\Sigma_{i}\right)\right\} \stackrel{L}{\rightarrow} N\left(0, \Pi_{i}\right),
$$


where $\Pi_{i}$ will be given explicitly in Section $2^{4}$. Moreover, defining $x_{j, i}=\operatorname{vech}\left(y_{j, i} y_{j, i}^{\prime}\right)$ and

$$
G_{i}=\sum_{j=1}^{M} x_{j, i} x_{j, i}^{\prime}-\frac{1}{2} \sum_{j=1}^{M-1}\left(x_{j, i} x_{j+1, i}^{\prime}+x_{j+1, i} x_{j, i}^{\prime}\right)
$$

we have that $M \hbar^{-1} G_{i}$, which is positive semi-definite, converges in probability to $\Pi_{i}$. Unconditionally this yields a feasible, mixed Gaussian limit theory. Further, the joint limit theory for $\left[y_{M}^{*}\right]_{i}$ and $\left[y_{M}^{*}\right]_{i^{\prime}}$, for $i \neq i^{\prime}$, is block diagonal allowing easy testing of the stability of aspects of the covariance structure of returns.

- We explore the accuracy of this feasible limit theory in a Monte Carlo study. In the univariate case this theory specialises to

$$
\frac{\left\{\left[y_{M}^{*}\right]_{i}-\sum_{i}\right\}}{\sqrt{\sum_{j=1}^{M} y_{j, i}^{4}-\sum_{j=2}^{M} y_{j, i}^{2} y_{j+1, i}^{2}}} \stackrel{L}{\rightarrow} N(0,1)
$$

which is asymptotically equivalent to (15) as

$$
\frac{M}{\hbar}\left\{\sum_{j=1}^{M} y_{j, i}^{4}-\sum_{j=2}^{M} y_{j, i}^{2} y_{j+1, i}^{2}\right\} \stackrel{p}{\rightarrow} 2 \int_{(i-1) \hbar}^{i \hbar} \Sigma^{2}(u) \mathrm{d} u
$$

in that case.

- In the bivariate case we derive the asymptotic distribution of the realised regression estimator. When we take one of the assets as a market portfolio we can regard the regression as a high frequency estimate of a beta.

- We derive the asymptotic distribution of the realised correlation between two assets. Realised correlations have been previously studied empirically by Andersen, Bollerslev, Diebold, and Labys (2001b) but no distributional theory has been available to allow us to assess the precision of the realised correlations.

The structure of the paper is as follows. In Section 2 we will give two theorems concerning the asymptotic distribution of realised covariation. In Section 3 we transform the asymptotic theory to give us a theory for measuring correlation and performing regression using high frequency data. Section 4 performs some Monte Carlo experiments to assess the accuracy of the theory for finite values of $M$. This section also studies the effect of leverage on our results. The overall conclusion from this work is that our theory seems entirely robust to leverage. However proving this result seems very challenging. Section 5 illustrates the use of this theory on some financial data. Section 6 concludes, while there is an Appendix which contains the proofs of the theorems given in the paper.

\footnotetext{
${ }^{4}$ Recall the vech notation stacks the (unique) lower triangular elements of the columns of a matrix into a vector. See, for example, Lutkepohl (1996, Ch. 7).
} 


\section{Theoretical framework}

\subsection{Multivariate SV model}

We consider $q$ stochastic processes $y_{\nu}^{*}(\nu=1, \ldots, q)$, determined by a set of stochastic differential equations

$$
\mathrm{d} y_{\nu}^{*}(t)=\mathrm{d} \alpha_{\nu}^{*}(t)+\sum_{a=1}^{m} \gamma_{\nu}^{a}(u) \mathrm{d} w_{a}(u)
$$

with initial condition $y_{\nu}^{*}(0)=0$. Using this notation the spot covolatility matrix of the SV model is

$$
\Sigma_{k l}(t)=\sum_{a=1}^{m} \gamma_{k}^{a}(t) \gamma_{l}^{a}(t) .
$$

This demonstrates that there is no loss in generality in moving from the SV model (13) to the form of (21).

Here we are making the following assumptions

- $\alpha_{\nu}^{*}$ and $\gamma_{\nu}^{a}>0(a=1, \ldots, m)$ are processes of locally bounded variation.

- $w=\left(w_{1}, \ldots, w_{m}\right)$ is $m$-dimensional standard Brownian motion (here $m$ could be bigger, equal to or smaller than $q$ ).

- The families of processes $\alpha_{\nu}$ and $\gamma_{\nu}^{a}$ are assumed to be jointly independent of $w$.

- $\alpha_{\nu}^{*}$ satisfies assumption $(\mathbf{A})$ given above.

For $j=1, \ldots, M$, let

$$
y_{j, i(\nu)}=y_{\nu}^{*}\left\{(i-1) \hbar+j \hbar M^{-1}\right\}-y_{\nu}^{*}\left\{(i-1) \hbar+(j-1) \hbar M^{-1}\right\}
$$

and

$$
\alpha_{j, i(\nu)}=\alpha_{\nu}^{*}\left\{(i-1) \hbar+j \hbar M^{-1}\right\}-\alpha_{\nu}^{*}\left\{(i-1) \hbar+(j-1) \hbar M^{-1}\right\}
$$

while

$$
\alpha_{\nu, i}=\alpha_{\nu}^{*}(i \hbar)-\alpha_{\nu}^{*}((i-1) \hbar)
$$

and

$$
\gamma_{\nu, i}^{a}=\int_{\hbar(i-1)}^{\hbar i} \gamma_{\nu}^{a}(u) \mathrm{d} u .
$$

In order to simplify the notation, we will drop all mention of the subscript $i$ in this and the next section as its role is entirely passive for it is fixed in this analysis. Hence, for example $y_{j, i(\nu)}$ and $\gamma_{\nu, i}^{a}$ become $y_{j(\nu)}$ and $\gamma_{\nu}^{a}$ respectively. But note that with this temporary convention $\gamma_{\nu}^{a}$ does not stand for the process with sample path $\gamma_{\nu}^{a}(t)$. 


\section{$2.2 \quad$ Results}

We shall refer to condition (A) given above in (12). Then we have three main results, the proofs of which are given in the Appendix.

Theorem 1 Suppose condition (A) is satisfied. Conditionally on

$$
\left\{\alpha_{\nu}, \gamma_{\nu}^{a}\right\}_{\nu=1, \ldots, q ; a=1, \ldots, m}
$$

the realised covariation matrix

$$
\left[y_{M}^{*}\right]=\left\{\sum_{j=1}^{M} y_{j(k)} y_{j(l)}\right\}_{k, l=1,2, \ldots, q}
$$

follows asymptotically, as $M \rightarrow \infty$, the normal law with mean $\Sigma$ and covariance matrix $\left(\hbar M^{-1}\right) \Omega$ where $\Sigma$ is the $q \times q$ matrix with

$$
\Sigma=\int_{\hbar(i-1)}^{\hbar i} \Sigma(u) \mathrm{d} u=\left\{\int_{\hbar(i-1)}^{\hbar i} \Sigma_{k l}(u) \mathrm{d} u\right\}_{k, l=1, \ldots, q}
$$

and $\Omega$ is the $q^{2} \times q^{2}$ array with elements

$$
\Omega=\left\{\int_{\hbar(i-1)}^{\hbar i}\left\{\Sigma_{k k^{\prime}}(u) \Sigma_{l l^{\prime}}(u)+\Sigma_{k l^{\prime}}(u) \Sigma_{l k^{\prime}}(u)\right\} \mathrm{d} u\right\}_{k, k^{\prime}, l, l^{\prime}=1, \ldots, q} .
$$

Corollary 1 Unconditionally the asymptotic law of

$$
\sqrt{\frac{M}{\hbar}}\left(\left[y_{M}^{*}\right]-\Sigma\right)
$$

is mixed normal with mean 0 and random covariance matrix $\Omega$.

This is an important result. In particular

- The rate of convergence is $\sqrt{M}$ for all components of the realised covariation.

- No kowledge of the drift process or spot covariance matrix is needed to use this theory.

- The limit theorem is mixed Gaussian, that is $\Omega$ is a stochastic matrix. This means that the difference between realised covariation matrix and actual covariation matrix will be heavier tailed than Gaussian. 
- The size of realised covariation matrix errors depends upon the level of volatility of the process. This impacts not just the precision of the realised variance but also the realised covariances.

- Inevitably $\Omega$ is singular, due to the symmetric nature of $\left[y_{M}^{*}\right]$ and $\Sigma$. Later we will write the theory in terms of the unique elements of these matrices, by employing vech transformations, however for the moment we prefer to maintain the general structure for this makes the proof more straightforward as it can be carried out using standard tensor notation.

Unfortunately $\Omega$ is not known and so this result is infeasible. However, the following theorem means that $\Omega$ can be replaced by a consistent estimator, thus providing a feasible theory.

Theorem 2 Suppose condition (A) is satisfied. Then let

$$
\widehat{\psi}_{k l k^{\prime} l^{\prime}}=\frac{M}{\hbar} \sum_{j=1}^{M} y_{j(k)} y_{j(l)} y_{j\left(k^{\prime}\right)} y_{j\left(l^{\prime}\right)}
$$

and

$$
\widetilde{\psi}_{k l k^{\prime} l^{\prime}}=\frac{M}{\hbar} \sum_{j=1}^{M-1} y_{j(k)} y_{j(l)} y_{j+1\left(k^{\prime}\right)} y_{j+1\left(l^{\prime}\right)} .
$$

Then

$$
\widehat{\psi}_{k l k^{\prime} l^{\prime}} \stackrel{p}{\rightarrow} \int_{\hbar(i-1)}^{\hbar i}\left\{\Sigma_{k k^{\prime}}(u) \Sigma_{l l^{\prime}}(u)+\Sigma_{k l^{\prime}}(u) \Sigma_{l k^{\prime}}(u)+\Sigma_{k l}(u) \Sigma_{k^{\prime} l^{\prime}}(u)\right\} \mathrm{d} u
$$

while

$$
\begin{aligned}
\widetilde{\psi}_{k l k^{\prime} l^{\prime}} & \stackrel{p}{\rightarrow} \int_{\hbar(i-1)}^{\hbar i} \Sigma_{k l}(u) \Sigma_{k^{\prime} l^{\prime}}(u) \mathrm{d} u \\
& =\underset{M \rightarrow \infty}{\mathrm{p}-\lim _{M \rightarrow \infty}} \widetilde{\psi}_{k^{\prime} l^{\prime} k l} .
\end{aligned}
$$

Corollary 2 Defining

$$
\bar{\psi}_{k l k^{\prime} l^{\prime}}=\widehat{\psi}_{k l k^{\prime} l^{\prime}}-\frac{1}{2}\left(\widetilde{\psi}_{k l k^{\prime} l^{\prime}}+\widetilde{\psi}_{k^{\prime} l^{\prime} k l}\right)
$$

we see that

$$
\bar{\psi}_{k l k^{\prime} l^{\prime}} \stackrel{p}{\rightarrow} \int_{\hbar(i-1)}^{\hbar i}\left\{\Sigma_{k k^{\prime}}(u) \Sigma_{l l^{\prime}}(u)+\Sigma_{k l^{\prime}}(u) \Sigma_{l k^{\prime}}(u)\right\} \mathrm{d} u,
$$

and so there exists a random $q^{2} \times q^{2}$ matrix

$$
H=\sum_{j=1}^{M} x_{j} x_{j}^{\prime}-\frac{1}{2} \sum_{j=1}^{M-1}\left(x_{j} x_{j+1}^{\prime}+x_{j+1} x_{j}^{\prime}\right),
$$

where $x_{j}=\operatorname{vec}\left(y_{j} y_{j}^{\prime}\right)$, explicitly calculable in terms of $y_{M}^{*}$, such that

$$
\frac{M}{\hbar} H \stackrel{p}{\rightarrow} \Omega
$$

as $M \rightarrow \infty$.

\footnotetext{
${ }^{5}$ Recall the vec notation stacks the columns of a matrix into a vector.
} 
The above corollary provides a general framework for the asymptotics for realised covariation. A convenient feature of the matrix $H$ in (31) is that it is positive semi-definite. This follows from the property of the first order serial correlation coefficient that its square is less than or equal to one. In particular the result follows because for any conformable vector $c$,

$$
c^{\prime} H c=\sum_{j=1}^{M}\left(c^{\prime} x_{j}\right)^{2}-\sum_{j=1}^{M-1}\left(c^{\prime} x_{j}\right)\left(c^{\prime} x_{j+1}\right) \geq 0 .
$$

In general $H$ will be singular as $\left[y_{M}^{*}\right]$ is a symmetric matrix ${ }^{6}$.

It is sometimes convenient to avoid the symmetric replication in the realised covariation matrix by employing a vech transformation. Then the limit theory can be easily written as

Corollary 3 As $M \rightarrow \infty$, conditionally

$$
\sqrt{\frac{M}{\hbar}}\left\{\operatorname{vech}\left(\left[y_{M}^{*}\right]\right)-\operatorname{vech}(\Sigma)\right\} \stackrel{L}{\rightarrow} N(0, \Pi),
$$

while, defining $x_{j}=\operatorname{vech}\left(y_{j} y_{j}^{\prime}\right)$ and

$$
G=\sum_{j=1}^{M} x_{j} x_{j}^{\prime}-\frac{1}{2} \sum_{j=1}^{M-1}\left(x_{j} x_{j+1}^{\prime}+x_{j+1} x_{j}^{\prime}\right)
$$

we have that

$$
\frac{M}{\hbar} G \stackrel{p}{\rightarrow} \Pi
$$

The matrix $G$ is still only guaranteed to be positive semi-definite, but should be positive definite in practice.

Finally we note the following result.

Corollary 4 The asymptotic unconditional covariance of the error term is, if it exists,

$$
\mathrm{E}(\Omega)=\left\{\int_{\hbar(i-1)}^{\hbar i}\left[\mathrm{E}\left\{\Sigma_{k k^{\prime}}(u) \Sigma_{l l^{\prime}}(u)\right\}+\mathrm{E}\left\{\Sigma_{k l^{\prime}}(u) \Sigma_{l k^{\prime}}(u)\right\}\right] \mathrm{d} u\right\}_{k, k^{\prime}, l, l^{\prime}=1, \ldots, q} .
$$

Previously Meddahi (2002) has studied this covariance quantity, but using very different methods. Importantly he proved that this result is independent of a no leverage assumption in the case where volatility is a stationary diffusion. We will return to this point in Section 4.3 of the paper.

\footnotetext{
${ }^{6}$ This argument is familiar in econometrics where Newey and West (1987) prove positive semi-definiteness of the Bartlett zero frequency spectral density estimator. Of course our analysis gives a much simpler result here.
} 


\subsection{Discussion}

The general results are compact. It is helpful to look at special cases in order to gain further understanding.

Example 1 Suppose we are interested in the joint distribution of realised covariation in the bivariate case. Then the Theorem 1 tells us that

$$
\begin{aligned}
& \sqrt{\frac{M}{\hbar}}\left\{\left(\begin{array}{l}
\sum_{j=1}^{M} y_{j(k)}^{2} \\
\sum_{j=1}^{M} y_{j(k)} y_{j(l)} \\
\sum_{j=1}^{M} y_{j(l)}^{2}
\end{array}\right)-\int_{\hbar(i-1)}^{\hbar i}\left(\begin{array}{c}
\Sigma_{k k}(u) \\
\Sigma_{k l}(u) \\
\Sigma_{l l}(u)
\end{array}\right) \mathrm{d} u\right\} \\
& \stackrel{L}{\rightarrow} N\left[0, \int_{\hbar(i-1)}^{\hbar i}\left\{\begin{array}{lll}
2 \Sigma_{k k}^{2}(u) & 2 \Sigma_{k k}(u) \Sigma_{k l}(u) & 2 \Sigma_{k l}^{2}(u) \\
2 \Sigma_{k k}(u) \Sigma_{k l}(u) & \Sigma_{k k}(u) \Sigma_{l l}(u)+\Sigma_{k l}^{2}(u) & 2 \Sigma_{l l}(u) \Sigma_{k l}(u) \\
2 \Sigma_{k l}^{2}(u) & 2 \Sigma_{l l}(u) \Sigma_{k l}(u) & 2 \Sigma_{l l}^{2}(u)
\end{array}\right\} \mathrm{d} u\right]
\end{aligned}
$$

The result on realised variances $\sum_{j=1}^{M} y_{j(k)}^{2}$ and $\sum_{j=1}^{M} y_{j(l)}^{2}$ were first derived in Barndorff-Nielsen and Shephard (2002a). The result on the marginal distribution of realised covariance as $M \rightarrow \infty$ is that

$$
\frac{\sqrt{\frac{M}{\hbar}}\left\{\sum_{j=1}^{M} y_{j(k)} y_{j(l)}-\int_{\hbar(i-1)}^{\hbar i} \Sigma_{k l}(u) \mathrm{d} u\right\}}{\sqrt{\int_{\hbar(i-1)}^{\hbar i}\left\{\Sigma_{k k}(u) \Sigma_{l l}(u)+\Sigma_{k l}^{2}(u)\right\} \mathrm{d} u}} \stackrel{L}{\rightarrow} N(0,1),
$$

which seems new, as does the joint distribution. Notice that when the spot correlation is zero then $\Sigma$ is diagonal. When $\Sigma_{k k}(t)=\Sigma_{l l}(t)$, then the asymptotic covariance becomes

$$
\int_{\hbar(i-1)}^{\hbar i} \Sigma_{k k}^{2}(s)\left\{\begin{array}{lll}
2 & 2 \rho_{k, l}(u) & 2 \rho_{k, l}^{2}(u) \\
2 \rho_{k, l}(u) & \left(1+\rho_{k, l}^{2}(u)\right) & 2 \rho_{k, l}(u) \\
2 \rho_{k, l}^{2}(u) & 2 \rho_{k, l}(u) & 2
\end{array}\right\} \mathrm{d} u
$$

where

$$
\rho_{k, l}(u)=\frac{\Sigma_{k l}(u)}{\Sigma_{k k}(u) \Sigma_{l l}(u)} .
$$

This last result is a generalisation of the result given in Anderson (1984, p. 121) on the asymptotic joint distribution in the case of i.i.d. Gaussian data.

Example 2 Special cases of Theorem 2 include

$$
\begin{gathered}
\frac{M}{\hbar} \sum_{j=1}^{M} y_{j(k)}^{4} \stackrel{p}{\rightarrow} 3 \int_{\hbar(i-1)}^{\hbar i} \Sigma_{k k}^{2}(u) \mathrm{d} u \\
\frac{M}{\hbar} \sum_{j=1}^{M} y_{j(k)}^{3} y_{j(l)} \stackrel{p}{\rightarrow} 3 \int_{\hbar(i-1)}^{\hbar i} \Sigma_{k k}(u) \Sigma_{k l}(u) \mathrm{d} u \\
\frac{M}{\hbar} \sum_{j=1}^{M} y_{j(k)}^{2} y_{j(l)}^{2} \stackrel{p}{\rightarrow} \int_{\hbar(i-1)}^{\hbar i}\left\{2 \Sigma_{k l}^{2}(u)+\Sigma_{k k}(u) \Sigma_{l l}(u)\right\} \mathrm{d} u .
\end{gathered}
$$


Likewise

$$
\begin{gathered}
\frac{M}{\hbar} \sum_{j=1}^{M} y_{j(k)}^{2} y_{j+1(l)}^{2} \stackrel{p}{\rightarrow} \int_{\hbar(i-1)}^{\hbar i} \Sigma_{k k}(u) \Sigma_{l l}(u) \mathrm{d} u, \\
\frac{M}{\hbar} \sum_{j=1}^{M}\left(y_{j(k)} y_{j(l)}\right)\left(y_{j+1(k)} y_{j+1(l)}\right) \stackrel{p}{\rightarrow} \int_{\hbar(i-1)}^{\hbar i} \Sigma_{k l}^{2}(u) \mathrm{d} u
\end{gathered}
$$

Example 3 Example 1 gives us an infeasible limit theorem, for the covariance matrix is stochastic and unknown. Theorem 2 provides a consistent estimator of it. In particular, examples of the theory include that

$$
\frac{\sum_{j=1}^{M} y_{j(k)}^{2}-\int_{\hbar(i-1)}^{\hbar i} \Sigma_{k k}(u) \mathrm{d} u}{\sqrt{\sum_{j=1}^{M} y_{j(k)}^{4}-\sum_{j=1}^{M-1} y_{j(k)}^{2} y_{j+1(k)}^{2}}} \stackrel{L}{\rightarrow} N(0,1)
$$

and

$$
\frac{\sum_{j=1}^{M} y_{j(k)} y_{j(l)}-\int_{\hbar(i-1)}^{\hbar i} \Sigma_{k l}(u) \mathrm{d} u}{\sqrt{\sum_{j=1}^{M} y_{j(k)}^{2} y_{j(l)}^{2}-\sum_{j=1}^{M-1} y_{j(k)} y_{j(l)} y_{j+1(k)} y_{j+1(l)}}} \stackrel{L}{\rightarrow} N(0,1) .
$$

The latter result seems new, the former is an asymptotically equivalent form of the result given in (15) due to Barndorff-Nielsen and Shephard (2002a) ${ }^{7}$. The denominators in both of these limit theorems are guaranteed to be non-negative by the property of the first order serial correlation coefficient. The results in the previous example suggest an asymptotically equivalent alternative to (48), that is

$$
\frac{\sum_{j=1}^{M} y_{j(k)} y_{j(l)}-\int_{\hbar(i-1)}^{\hbar i} \Sigma_{k l}(u) \mathrm{d} u}{\sqrt{\frac{1}{2}\left(\sum_{j=1}^{M} y_{j(k)}^{2} y_{j(l)}^{2}+\sum_{j=1}^{M-1} y_{j(k)}^{2} y_{j+1(l)}^{2}\right)}} \stackrel{L}{\rightarrow} N(0,1) .
$$

\section{Asymptotic theory for regression and correlation}

\subsection{Regression}

Regression plays a central role both in theoretical and empirical financial economics (e.g. see Cochrane (2001, Ch. 12) and Campbell, Lo, and MacKinlay (1997, Ch. 5) which are both devoted to regression). For example, the regression of the returns of an individual asset on a wide market index is often called a "beta" (e.g. Lintner (1965)). In this Section we use our distribution theory for realised covariation to derive a theory for univariate regression. Again this for

${ }^{7}$ The replacement of $\frac{2}{3} \sum_{j=1}^{M} y_{j(k)}^{4}$ by $\sum_{j=1}^{M} y_{j(k)}^{4}-\sum_{j=2}^{M} y_{j(k)}^{2} y_{j+1(k)}^{2}$ leaves the asymptotic result unchanged

$$
M \sum_{j=1}^{M} y_{j(k)}^{4} \stackrel{p}{\rightarrow} 3 \int_{\hbar(i-1)}^{\hbar i} \Sigma_{k k}^{2}(s) \mathrm{d} s
$$

and

$$
M \sum_{j=1}^{M} y_{j(k)}^{2} y_{j+1(k)}^{2} \stackrel{p}{\rightarrow} \int_{\hbar(i-1)}^{\hbar i} \Sigma_{k k}^{2}(s) \mathrm{d} s .
$$


will be based on fixed intervals of time and allowing the number of high frequency observations to go to infinity within that interval. We regress variable $l$ on variable $k$, then again surpressing subscripts $i$,

$$
\widehat{\beta}_{(k l)}=\frac{\sum_{j=1}^{M} y_{j(k)} y_{j(l)}}{\sum_{j=1}^{M} y_{j(k)}^{2}} .
$$

This involves just elements of the realised covariation and so we can use the asymptotic theory of the previous section to derive its asymptotic distribution. The probability limit of regression is known by the theory of QV. In particular

$$
\widehat{\beta}_{(l k)} \stackrel{p}{\rightarrow} \frac{\left[y_{(k)}^{*}, y_{(l)}^{*}\right]}{\left[y_{(k)}^{*}\right]}=\beta_{(l k)},
$$

a result which is discussed at some length in, for example, Back (1991) and Andersen, Bollerslev, Diebold, and Labys (2001b). Empirical estimates of regression parameters using high frequency data have been computed by, for example, Andersen, Bollerslev, Diebold, and Ebens (2001) and Liu (2002). Here we extend the theoretical results to derive the asymptotic distribution, under our additional assumptions given above. In this case $\beta_{(l k)}$ has the simpler form of

$$
\beta_{(l k)}=\frac{\int_{\hbar(i-1)}^{\hbar i} \Sigma_{k l}(u) \mathrm{d} u}{\int_{\hbar(i-1)}^{\hbar i} \Sigma_{k k}(u) \mathrm{d} u}
$$

The asymptotic distribution can be derived using standard linearisation methods ${ }^{8}$, which approximates $\sqrt{\frac{M}{\hbar}}\left(\widehat{\beta}_{(l k)}-\beta_{(l k)}\right)$ by

$$
\frac{\sum_{j=1}^{M} y_{j(k)} y_{j(l)}-\beta_{(l k)} \sum_{j=1}^{M} y_{j(k)}^{2}}{\int_{\hbar(i-1)}^{\hbar i} \Sigma_{k k}(u) \mathrm{d} u} .
$$

It yields the result

Proposition 1 Define

$$
g_{(l k)}=d_{(l k)}^{\prime} \Psi_{(l k)} d_{(l k)}
$$

where

$$
\Psi_{(l k)}=\int_{\hbar(i-1)}^{\hbar i}\left\{\begin{array}{ll}
\Sigma_{k k}(u) \Sigma_{l l}(u)+\Sigma_{k l}^{2}(u) & 2 \Sigma_{k k}(u) \Sigma_{k l}(u) \\
2 \Sigma_{k k}(u) \Sigma_{k l}(u) & 2 \Sigma_{k k}^{2}(u)
\end{array}\right\} \mathrm{d} u \quad \text { and } \quad d_{(l k)}=\left(\begin{array}{c}
1 \\
-\beta_{(l k)}
\end{array}\right) .
$$

\footnotetext{
${ }^{8}$ This is based on approximating $x / y$ by

$$
\begin{aligned}
& \frac{\mu_{x}}{\mu_{y}}+\frac{x-\mu_{x}}{\mu_{y}}-\frac{\left(y-\mu_{y}\right) \mu_{x}}{\mu_{y}^{2}} \\
= & \frac{\mu_{x}}{\mu_{y}}+\frac{x}{\mu_{y}}-\frac{y \mu_{x}}{\mu_{y}^{2}},
\end{aligned}
$$
}

where $\mu_{x}$ and $\mu_{y}$ are the p-lims of $x$ and $y$ respectively. 
Then as $M \rightarrow \infty$ so

$$
\frac{\sqrt{\frac{M}{\hbar}}\left(\widehat{\beta}_{(l k)}-\beta_{(l k)}\right)}{\sqrt{\left(\int_{\hbar(i-1)}^{\hbar i} \Sigma_{k k}(u) \mathrm{d} u\right)^{-2} g_{(l k)}}} \stackrel{L}{\rightarrow} N(0,1) .
$$

Example 4 When $\Sigma_{k l}(u)=0$ and so $\beta_{(l k)}=0$, we have that

$$
\frac{\sqrt{\frac{M}{\hbar}} \widehat{\beta}_{(l k)}}{\sqrt{\left(\int_{\hbar(i-1)}^{\hbar i} \Sigma_{k k}(u) \mathrm{d} u\right)^{-2} \int_{\hbar(i-1)}^{\hbar i} \Sigma_{k k}(u) \Sigma_{l l}(u) \mathrm{d} u}} \stackrel{L}{\rightarrow} N(0,1)
$$

In practice we have to replace $\Psi_{(l k)}$ and $d_{(l k)}$ by estimators to make the above regression theory feasible. However, the Theorem 2 from the previous section implies this is straightforward. In particular

Proposition 2 Define

$$
x_{j}=y_{j(k)} y_{j(l)}-\widehat{\beta}_{(l k)} y_{j(k)}^{2}
$$

and

$$
\widehat{g}_{(l k)}=\sum_{j=1}^{M} x_{j}^{2}-\sum_{j=1}^{M-1} x_{j} x_{j+1}
$$

Then as $M \rightarrow \infty$ so

$$
\frac{\widehat{\beta}_{(l k)}-\beta_{(l k)}}{\sqrt{\left(\sum_{j=1}^{M} y_{j(k)}^{2}\right)^{-2} \widehat{g}_{(l k)}}} \stackrel{L}{\rightarrow} N(0,1) .
$$

An attractive feature of this theory is that all of the required terms are straightforward to compute. It is interesting to note that $\sum_{j=1}^{M} x_{j}=0$ exactly in this context.

\subsection{Correlation}

The same strategy can be used to derive the asymptotic distribution of the realised correlation coefficient. We define

$$
\widehat{\rho}_{(l k)}=\frac{\sum_{j=1}^{M} y_{j(k)} y_{j(l)}}{\sqrt{\sum_{j=1}^{M} y_{j(k)}^{2} \sum_{j=1}^{M} y_{j(l)}^{2}}} .
$$

The probability limit of the correlation is known by the theory of QV. In particular

$$
\widehat{\rho}_{(l k)} \stackrel{p}{\rightarrow} \frac{\left[y_{(k)}^{*}, y_{(l)}^{*}\right]}{\sqrt{\left[y_{(k)}^{*}\right]\left[y_{(l)}^{*}\right]}}=\rho_{(l k)},
$$

a result which is discussed at some length in Andersen, Bollerslev, Diebold, and Labys (2001b). Here we extend this result to derive the asymptotic distribution, under our additional assumptions given above. In this case

$$
\rho_{(l k)}=\frac{\int_{\hbar(i-1)}^{\hbar i} \Sigma_{k l}(u) \mathrm{d} u}{\sqrt{\int_{\hbar(i-1)}^{\hbar i} \Sigma_{k k}(u) \mathrm{d} u \int_{\hbar(i-1)}^{\hbar i} \Sigma_{l l}(u) \mathrm{d} u}} .
$$


The asymptotic distribution can be derived using standard linearisation methods ${ }^{9}$, which approximate $\sqrt{\frac{M}{\hbar}}\left(\widehat{\rho}_{(l k)}-\rho_{(l k)}\right)$ by

$$
\begin{aligned}
& \rho_{(l k)}\left(\frac{\sum_{j=1}^{M} y_{j(k)} y_{j(l)}}{\int_{\hbar(i-1)}^{\hbar i} \Sigma_{k l}(u) \mathrm{d} u}-\frac{1}{2} \frac{\sum_{j=1}^{M} y_{j(k)}^{2}}{\int_{\hbar(i-1)}^{\hbar i} \Sigma_{k k}(u) \mathrm{d} u}-\frac{1}{2} \frac{\sum_{j=1}^{M} y_{j(l)}^{2}}{\int_{\hbar(i-1)}^{\hbar i} \Sigma_{l l}(u) \mathrm{d} u}\right) \\
= & \frac{\sum_{j=1}^{M} y_{j(k)} y_{j(l)}-\frac{1}{2} \beta_{(l k)} \sum_{j=1}^{M} y_{j(k)}^{2}-\frac{1}{2} \beta_{(k l)} \sum_{j=1}^{M} y_{j(l)}^{2}}{\sqrt{\int_{\hbar(i-1)}^{\hbar i} \Sigma_{k k}(u) \mathrm{d} u \int_{\hbar(i-1)}^{\hbar i} \Sigma_{l l}(u) \mathrm{d} u}} .
\end{aligned}
$$

It yields the infeasible result

Proposition 3 Define

$$
g_{(l k)}=d_{(l k)}^{\prime} \Pi_{(l k)} d_{(l k)}
$$

where

$$
\Pi_{(l k)}=\int_{\hbar(i-1)}^{\hbar i}\left\{\begin{array}{lll}
2 \Sigma_{k k}^{2}(u) & 2 \Sigma_{k k}(u) \Sigma_{k l}(u) & 2 \Sigma_{k l}^{2}(u) \\
2 \Sigma_{k k}(u) \Sigma_{k l}(u) & \Sigma_{k k}(u) \Sigma_{l l}(u)+\Sigma_{k l}^{2}(u) & 2 \Sigma_{l l}(u) \Sigma_{k l}(u) \\
2 \Sigma_{k 2}^{2}(u) & 2 \Sigma_{l l}(u) \Sigma_{k l}(u) & 2 \Sigma_{l l}^{2}(u)
\end{array}\right\} \mathrm{d} u
$$

and

$$
d_{(l k)}=\left(\begin{array}{l}
-\frac{1}{2} \beta_{(l k)} \\
1 \\
-\frac{1}{2} \beta_{(k l)}
\end{array}\right)
$$

Here $\beta_{(l k)}$ is the population regression of the $l$-th variable on the $k$-th, while $\beta_{(k l)}$ is the regression of the $k$-th on the $l$-th. Then as $M \rightarrow \infty$ so

$$
\frac{\sqrt{\frac{M}{\hbar}}\left(\widehat{\rho}_{(l k)}-\rho_{(l k)}\right)}{\sqrt{\left(\int_{\hbar(i-1)}^{\hbar i} \Sigma_{k k}(u) \mathrm{d} u \int_{\hbar(i-1)}^{\hbar i} \Sigma_{l l}(u) \mathrm{d} u\right)^{-1} g_{i(l, k)}}} \stackrel{L}{\rightarrow} N(0,1) .
$$

The following example illustrates the theory.

Example 5 When $\Sigma_{k l}(s)=0$, we have that $\beta_{(l k)}=\beta_{(k l)}=0$ and $\rho_{(l k)}=0$ so

$$
\frac{\sqrt{\frac{M}{\hbar}} \widehat{\rho}_{(l k)}}{\sqrt{\left(\int_{\hbar(i-1)}^{\hbar i} \Sigma_{k k}(u) \mathrm{d} u \int_{\hbar(i-1)}^{\hbar i} \Sigma_{l l}(u) \mathrm{d} u\right)^{-1}\left(\int_{\hbar(i-1)}^{\hbar i} \Sigma_{k k}(u) \Sigma_{l l}(u) \mathrm{d} u\right)}} \stackrel{L}{\rightarrow} N(0,1) .
$$

This has quite a stable asymptotic variance, compared to the corresponding result for the regression coefficient (57), for it is unaffected by the scaling of any of the assets. Hence we might expect the variance to be less influenced by changes in the integrated variance of one of the assets.

${ }^{9}$ This is based on approximating $x / \sqrt{y z}$ by

$$
\begin{aligned}
& \frac{\mu_{x}}{\sqrt{\mu_{y} \mu_{z}}}+\frac{\left(x-\mu_{x}\right)}{\sqrt{\mu_{y} \mu_{z}}}-\frac{1}{2} \frac{\left(y-\mu_{y}\right) \mu_{x}}{\mu_{y} \sqrt{\mu_{y} \mu_{z}}}-\frac{1}{2} \frac{\left(z-\mu_{z}\right) \mu_{x}}{\mu_{z} \sqrt{\mu_{y} \mu_{z}}} \\
= & \frac{\mu_{x}}{\sqrt{\mu_{y} \mu_{z}}}+\rho\left(\frac{x}{\mu_{x}}-\frac{1}{2} \frac{y}{\mu_{y}}-\frac{1}{2} \frac{z}{\mu_{z}}\right),
\end{aligned}
$$

where $\mu_{x}, \mu_{y}, \mu_{z}$ are the p-lims of $x, y$ and $z$ respectively. 
Proposition 4 The feasible limit theory involves

$$
\begin{aligned}
x_{j} & =y_{j(k)} y_{j(l)}-\frac{1}{2} \widehat{\beta}_{(l k)} y_{j(k)}^{2}-\frac{1}{2} \widehat{\beta}_{(k l)} y_{j(l)}^{2} \\
& =\frac{1}{2} y_{j(k)}\left(y_{j(l)}-\widehat{\beta}_{(l k)} y_{j(k)}\right)+\frac{1}{2} y_{j(l)}\left(y_{j(k)}-\widehat{\beta}_{(k l)} y_{j(l)}\right)
\end{aligned}
$$

and

$$
\widehat{g}_{(l k)}=\sum_{j=1}^{M} x_{j}^{2}-\sum_{j=1}^{M-1} x_{j} x_{j+1}
$$

Then as $M \rightarrow \infty$ so

$$
\frac{\widehat{\rho}_{(l k)}-\rho_{(l k)}}{\sqrt{\left(\sum_{j=1}^{M} y_{j(k)}^{2} \sum_{j=1}^{M} y_{j(l)}^{2}\right)^{-1} \widehat{g}_{(l k)}}} \stackrel{L}{\rightarrow} N(0,1) .
$$

We again note the simplicity of this result and that $\sum_{j=1}^{M} x_{j}=0$.

\section{Small sample effectiveness of the limit theory}

\subsection{Simulation design}

In this subsection we document some Monte Carlo experiments we have performed in order to assess the accuracy of the above theory for realised covariation. Throughout we work with a bivariate factor based stochastic volatility model. Some literature on this topic includes Diebold and Nerlove (1989), Meddahi and Renault (1996), Meddahi and Renault (2002), Pitt and Shephard (1999), Chib, Nardari, and Shephard (1999), Barndorff-Nielsen and Shephard (2001a, Section 6.5) and Hubalek and Nicolato (2001). The structure of the model will be that

$$
\mathrm{d} y^{*}(t)=(B \quad I) \mathrm{d} s^{*}(t)
$$

where $s^{*}$ is a vector of independent, zero mean, SV models. That is

$$
\mathrm{d} s_{j}^{*}(t)=\sigma_{j}(t) \mathrm{d} w_{j}(t), \quad j=1, \ldots, m,
$$

where $\sigma_{j}(t)$ are spot volatility processes and are constrained to be non-negative and $w_{j}$ are standard Brownian motions. Throughout all the volatilities and the Brownian motions are taken to be independent. This structure implies that

$$
\left(\begin{array}{cccc}
\gamma_{1}^{1}(t) & \gamma_{1}^{2}(t) & \cdots & \gamma_{1}^{m}(t) \\
\gamma_{2}^{1}(t) & \gamma_{2}^{2}(t) & & \vdots \\
\vdots & & & \\
\gamma_{q}^{1}(t) & & & \gamma_{q}^{m}(t)
\end{array}\right)=\left(\begin{array}{ll}
B & I
\end{array}\right) \operatorname{diag}\left\{\sigma_{1}(t), \ldots, \sigma_{m}(t)\right\}
$$

and that

$$
\Sigma(t)=(B \quad I) \operatorname{diag}\left\{\sigma_{1}^{2}(t), \ldots, \sigma_{m}^{2}(t)\right\}\left(\begin{array}{ll}
B & I
\end{array}\right)^{\prime} .
$$


In the experiments in the first two subsections (in our third subsection we will look at a diffusion based model) we will assume that each spot volatility $\sigma$ follows a non-Gaussian Ornstein-Uhlenbeck process. That is the volatility follows the solution to the SDE

$$
\mathrm{d} \sigma_{j}^{2}(t)=-\lambda_{j} \sigma_{j}^{2}(t) \mathrm{d} t+\mathrm{d} z_{j}\left(\lambda_{j} t\right)
$$

where $z(t)$ is a Lévy process with non-negative increments. These models have been developed in this context by Barndorff-Nielsen and Shephard (2001a). They have the advantage, from the point of view of illustrating our theory, that the volatility occasionally jumps. The rather unusual timing for $\mathrm{d} z_{j}\left(\lambda_{j} t\right)$ is chosen so that the marginal distribution of $\sigma_{j}^{2}(t)$ does not vary with $\lambda_{j}$. This eases our understanding of the parameters which index this model. Related work includes the affine models of Duffie, Pan, and Singleton (2000).

In the Monte Carlo experiments we report we design (75) to have marginal distribution which is $\Gamma\left(\nu_{j}, a_{j}\right)$, while taking $\hbar=1$. Then our choices for the parameters will be

$$
B=\left(\begin{array}{l}
1 \\
1
\end{array}\right), \quad\left(\begin{array}{l}
\nu_{1} \\
\nu_{2} \\
\nu_{3}
\end{array}\right)=\left(\begin{array}{l}
2.0 \\
1.0 \\
1.5
\end{array}\right), \quad\left(\begin{array}{l}
a_{1} \\
a_{2} \\
a_{3}
\end{array}\right)=\left(\begin{array}{l}
5.0 \\
1.0 \\
1.0
\end{array}\right), \quad\left(\begin{array}{l}
\lambda_{1} \\
\lambda_{2} \\
\lambda_{3}
\end{array}\right)=\left(\begin{array}{l}
0.04 \\
0.13 \\
3.00
\end{array}\right) .
$$

This means that

$$
\mathrm{E}\left(\begin{array}{l}
\sigma_{1}^{2}(t) \\
\sigma_{2}^{2}(t) \\
\sigma_{3}^{2}(t)
\end{array}\right)=\left(\begin{array}{l}
0.4 \\
1.0 \\
1.5
\end{array}\right), \quad \operatorname{Cov}\left(\begin{array}{c}
\sigma_{1}^{2}(t) \\
\sigma_{2}^{2}(t) \\
\sigma_{3}^{2}(t)
\end{array}\right)=\left(\begin{array}{ccc}
0.02 & 0 & 0 \\
0 & 1.0 & 0 \\
0 & 0 & 1.5
\end{array}\right)
$$

which implies that the effect of the common component in the asset prices model $(74), \sigma_{1}^{2}(t)$, is small in comparison with the individual effects $\sigma_{2}^{2}(t)$ and $\sigma_{3}^{2}(t)$.

Figure 1 shows a short sample path from (74) using $M=72$, simulating 200 complete days. The top left graph in the figure shows the daily returns from the factor model. It is difficult to pick up any patterns in the data by looking at this data. The top right and middle right graphs show the realised variances for the two assets:

$$
\sum_{j=1}^{M} y_{j, i(1)}^{2} \text { and } \sum_{j=1}^{M} y_{j, i(2)}^{2}
$$

graphed against $i$, their corresponding day (recall the notation $i$ was surpressed in the previous two sections). Also drawn are there corresponding population versions $\int_{\hbar(i-1)}^{\hbar i} \Sigma_{11}(u) \mathrm{d} u$ and $\int_{\hbar(i-1)}^{\hbar i} \Sigma_{22}(u) \mathrm{d} u$. For asset 1 there is more memory in the volatility clustering, but it has significant upwards and downwards shifts. For asset 2 the series has only a small degree of memory.

The middle left graph in Figure 1 depicts the realised covariance $\sum_{j=1}^{M} y_{j, i(1)} y_{j, i(2)}$ of the series and shows it is quite a noisy estimator of the underlying actual covariance $\int_{\hbar(i-1)}^{\hbar i} \Sigma_{12}(u) \mathrm{d} u$. 

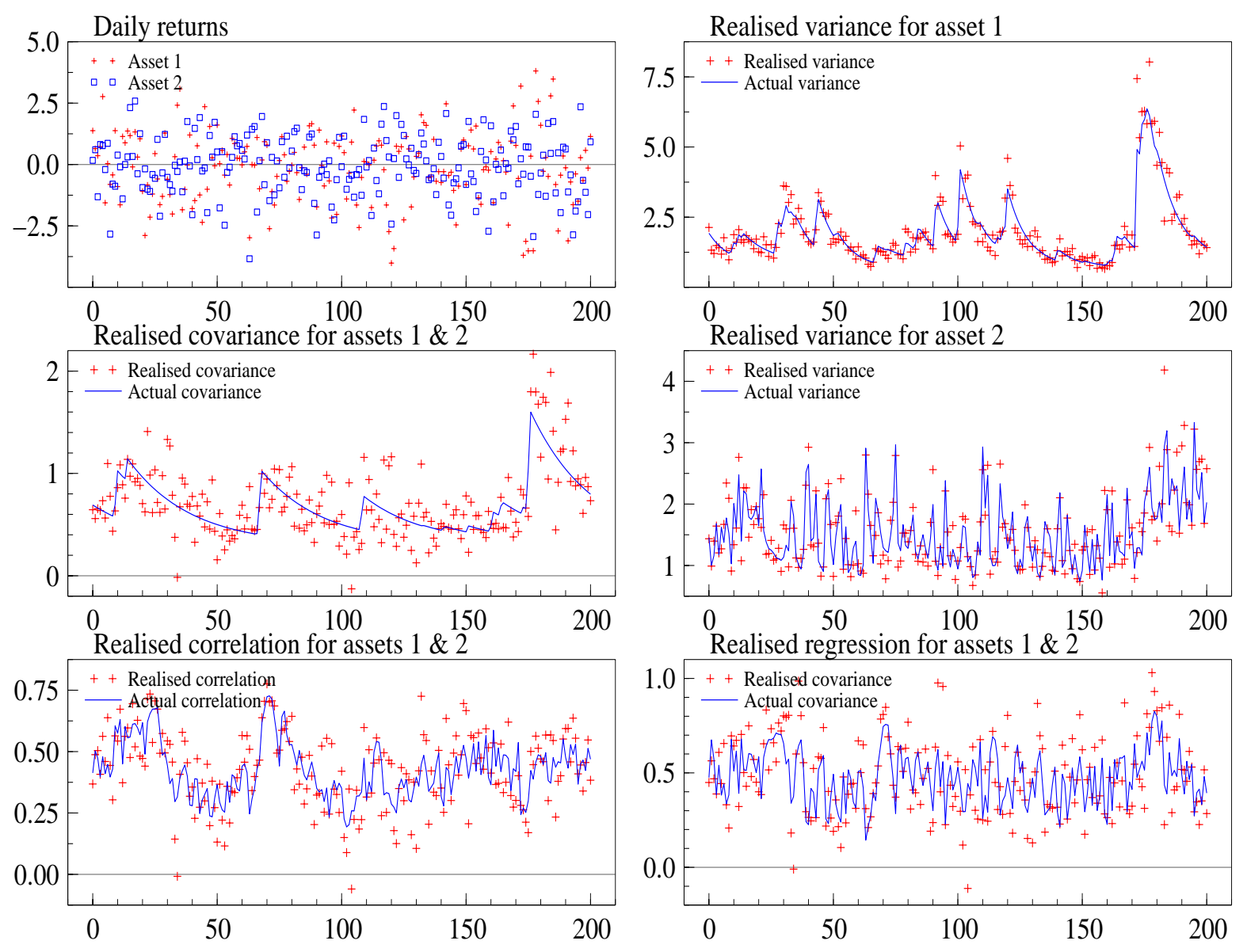

Figure 1: Simulation from a bivariate factor $S V$ model. Based on $M=72$ throughout. Top left: simulated daily returns. Top right: realised variance and actual variance for asset 1. Middle right: realised variance and actual variance for asset 2. Middle left: realised covariance and actual covariance for assets 1 \& 2. Bottom left: realised correlation and actual correlation between assets 1 \& 2. Bottom right: realised regression of asset 1 on asset 2, and actual regression. Code is available at: sim_mult.ox

The dependence structure of the data is much clearer in the bottom left graph which draws the realised correlation

$$
\frac{\sum_{j=1}^{M} y_{j, i(1)} y_{j, i(2)}}{\sqrt{\sum_{j=1}^{M} y_{j, i(1)}^{2} \sum_{j=1}^{M} y_{j, i(2)}^{2}}}
$$

amongst the two series. The errors in estimating the actual correlations

$$
\frac{\int_{\hbar(i-1)}^{\hbar i} \Sigma_{12}(u) \mathrm{d} u}{\sqrt{\int_{\hbar(i-1)}^{\hbar i} \Sigma_{11}(u) \mathrm{d} u \int_{\hbar(i-1)}^{\hbar i} \Sigma_{22}(u) \mathrm{d} u}}
$$

seem marginally more homoskedastic here, although when the correlation gets close to one their variance seems to get small. A less stable picture appears when one looks at the realised 
regression of asset 1 on asset 2 ,

$$
\frac{\sum_{j=1}^{M} y_{j, i(1)} y_{j, i(2)}}{\sum_{j=1}^{M} y_{j, i(2)}^{2}}
$$

which estimates

$$
\frac{\int_{\hbar(i-1)}^{\hbar i} \Sigma_{12}(u) \mathrm{d} u}{\int_{\hbar(i-1)}^{\hbar i} \Sigma_{22}(u) \mathrm{d} u}
$$

Here the magnitude of the difference between these two quantities seems to vary quite significantly through time.

In more extensive simulated data the above analysis showed that even with quite a large value of $M$ there are important differences between the realised quantities and the actual objects they estimate. Further, the properties of the errors seem to differ as we move from volatilities to covariances and on to correlations and correlations, with the errors being quite substantial when we study the desired realised correlations and regressions. Our asymptotic theory allows us to understand these objects. We now start using the theory to measure the size of the errors and use Monte Carlo results to indicate the accuracy of the asymptotic theory in finite sample cases.

\subsection{Assessing the performance of the asymptotic theory}

\subsubsection{Realised covariance}

Our asymptotic theory tells us that

$$
\frac{\sum_{j=1}^{M} y_{j, i(1)} y_{j, i(2)}-\int_{\hbar(i-1)}^{\hbar i} \Sigma_{12}(u) \mathrm{d} u}{\sqrt{\sum_{j=1}^{M} y_{j, i(1)}^{2} y_{j, i(2)}^{2}-\sum_{j=1}^{M-1} y_{j, i(1)} y_{j, i(2)} y_{j+1, i(1)} y_{j+1, i(2)}}} \stackrel{d}{\rightarrow} N(0,1) .
$$

How close to normality is this ratio for small and moderate values of $M$ ?

To investigate the effectiveness of the limit theory we have drawn in Figure 2 a plot of the realised covariance errors

$$
\sum_{j=1}^{M} y_{j, i(1)} y_{j, i(2)}-\int_{\hbar(i-1)}^{\hbar i} \Sigma_{21}(u) \mathrm{d} u
$$

against $i$ for the Monte Carlo design discussed in the previous subsection. As we move from the left hand side across the page we increase the value of $M$ and we can see the decrease in the spread of these errors. The Figure also gives $95 \%$ confidence intervals for the errors generated using the feasible limit theory (78). These also fall quite quickly with $M$ (theory tells us they fall at rate $\sqrt{M}$ ). An important feature of the confidence intervals is that they vary dramatically, sometimes being quite small, other times being large. This reflects the changing volatility in the series.

The coverage of the limit theory is assessed by the normal QQ plots given in the lower three plots in Figure 2. These are based on 2,000 simulated daily observations. These show quite 

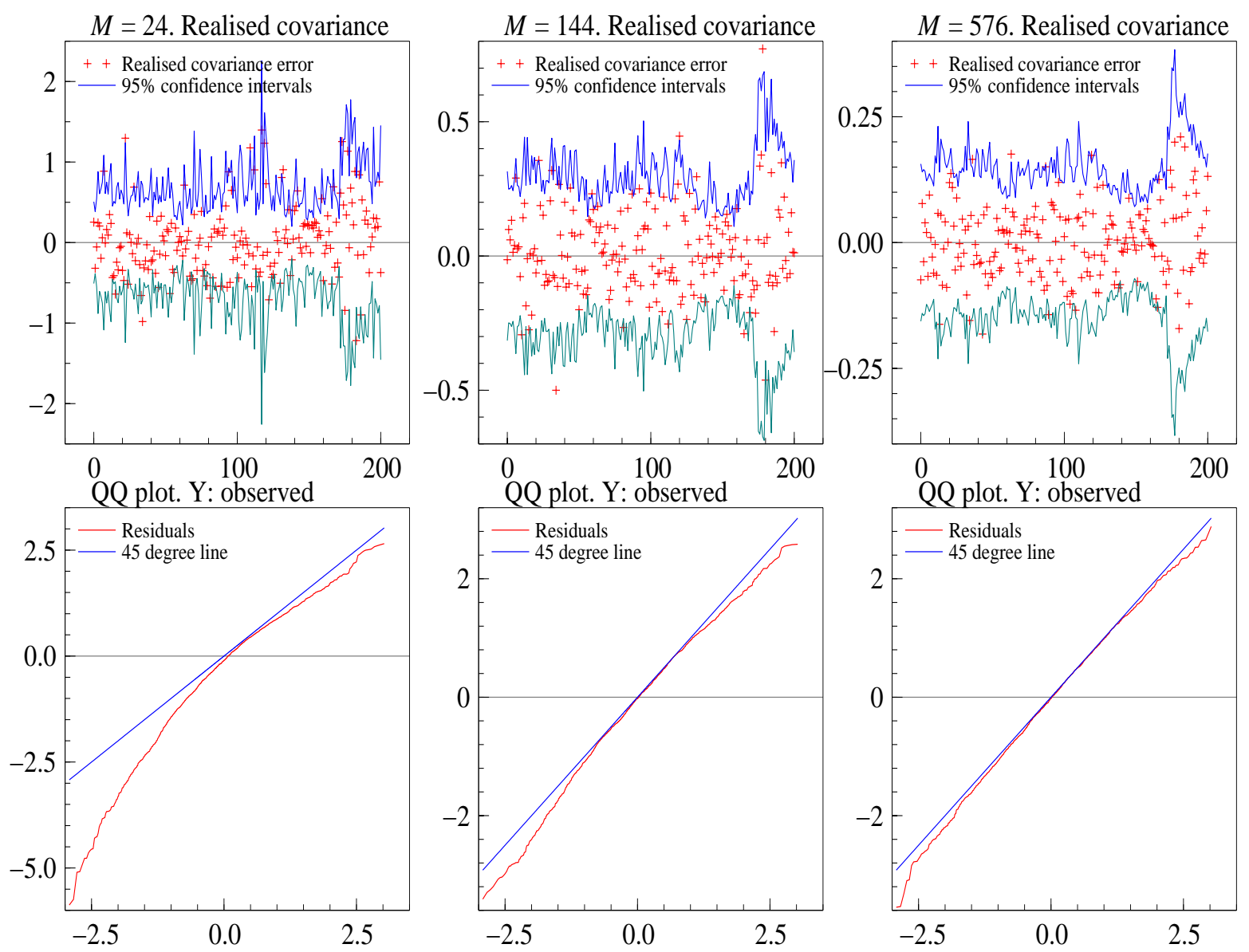

Figure 2: Simulation from a bivariate factor $S V$ model. Top line: drawn is the realised covariance errors and their 95\% confidence intervals. Bottom line are the normal QQ plots for the standardised errors. These plot on the y-axis the ranked observed standardised errors (which should be $N I D(0,1))$ against, on the x-axis, their expected quantities. Left to right increases $M$ through 24, 144 and 576. Code is available at: sim_mult.ox

poor results for small values of $M$, but with $M$ being large the limit theory can be seen to be effective.

\subsubsection{Realised regression}

Recall the limit theory for the regression of the returns of asset one on asset two. It has that as $M \rightarrow \infty$ so

$$
\frac{\widehat{\beta}_{i(12)}-\beta_{i(12)}}{\sqrt{\left(\sum_{j=1}^{M} y_{j, i(2)}^{2}\right)^{-2}\left\{\sum_{j=1}^{M} x_{j, i}^{2}-\sum_{j=1}^{M-1} x_{j, i} x_{j+1, i}\right\}}} \stackrel{L}{\rightarrow} N(0,1)
$$

where

$$
x_{j, i}=y_{j, i(1)} y_{j, i(2)}-\widehat{\beta}_{i(12)} y_{j, i(2)}^{2} .
$$


It is important to note that the returns on the two assets appear asymmetrically here, with the returns of the second asset appearing much more prominently. This means that if the first and second assets are rescaled by different amounts the regression coefficients and their standard errors change. However, unlike the realised covariance case, when both assets are rescaled by the same amount, the coefficients and the standard errors do not now change.
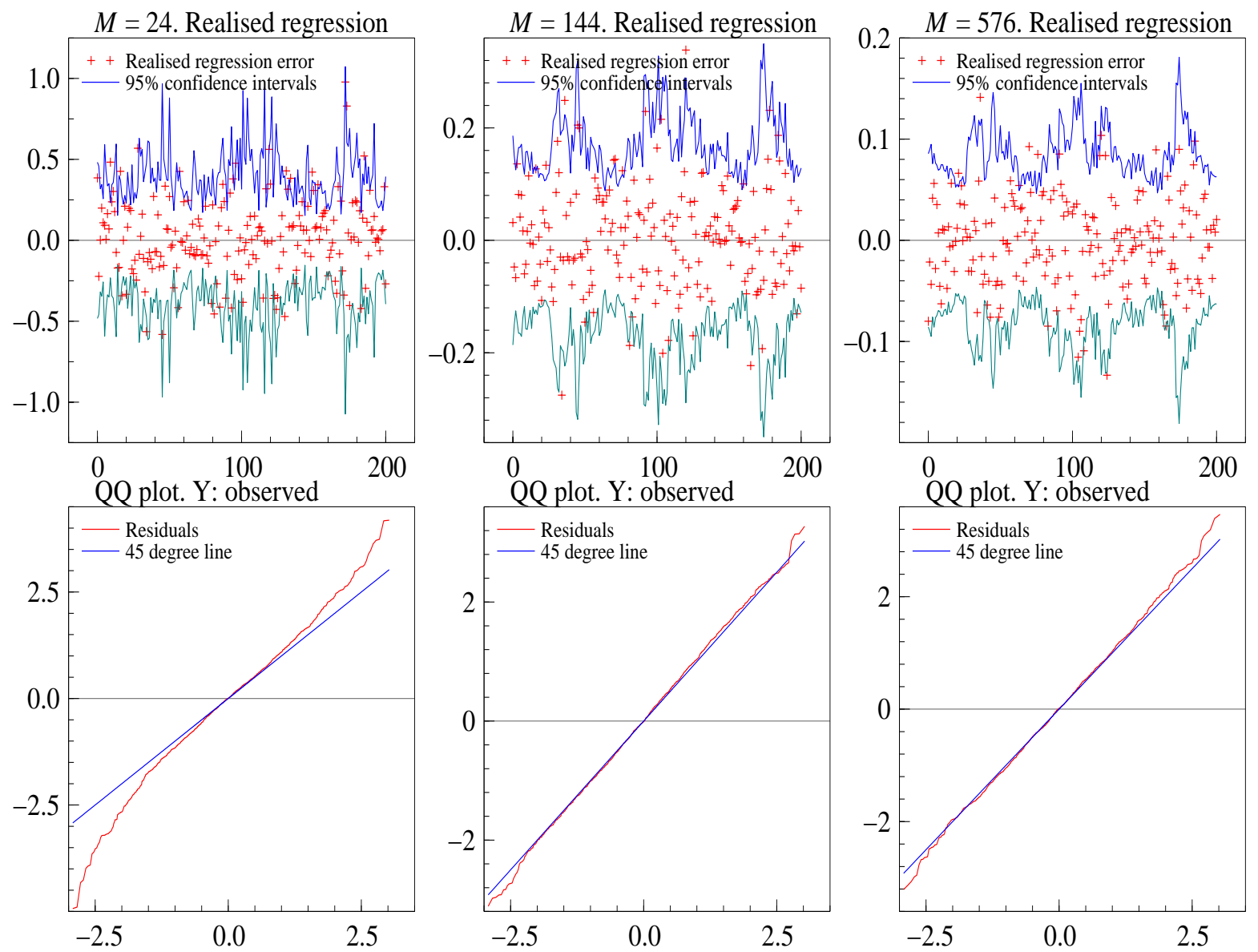

Figure 3: Simulation from a bivariate factor $S V$ model. Top line: drawn is the realised regression of asset one on asset two. Bottom line are the corresponding QQ plots. Code is available at: sim_mult.ox

The graphs on the top half of Figure 3 shows the regression errors

$$
\frac{\sum_{j=1}^{M} y_{j, i(1)} y_{j, i(2)}}{\sum_{j=1}^{M} y_{j, i(2)}^{2}}-\frac{\int_{\hbar(i-1)}^{\hbar i} \Sigma_{21}(u) \mathrm{d} u}{\int_{\hbar(i-1)}^{\hbar i} \Sigma_{22}(u) \mathrm{d} u},
$$

plotted against time together with asymptotically valid $95 \%$ confidence intervals for these errors based on (79). These Figures are computed for a variety of values of $M$. They show that the confidence intervals are more stable through time than was the case for the realised covariance. This is caused by the stablising $\left(\sum_{j=1}^{M} y_{j, i(2)}^{2}\right)^{-2}$ which appears in the denominator of (79). 
This means it is less sensitive to changes in the volatility of the second asset and so more simply reflects the dependence between the two assets than the covariance.

Figure 3 shows the corresponding QQ plots for the standardised regression errors. They are again based on 2,000 simulations and indicate that the asymptotic theory provides a reasonable basis for confidence intervals by the time $M$ is beyond 100. For smaller values of $M$ there are some significant distortions.

\subsubsection{Realised correlation}

The feasible limit theory for correlation of the returns of asset one on asset two is

$$
\frac{\widehat{\rho}_{i(12)}-\rho_{i(12)}}{\sqrt{\left(\sum_{j=1}^{M} y_{j, i(1)}^{2} \sum_{j=1}^{M} y_{j, i(2)}^{2}\right)^{-1}\left(\sum_{j=1}^{M} x_{j, i}^{2}-\sum_{j=1}^{M-1} x_{j, i} x_{j+1, i}\right)}} \stackrel{L}{\rightarrow} N(0,1),
$$

where

$$
x_{j, i}=y_{j, i(1)} y_{j, i(2)}-\frac{1}{2} \widehat{\beta}_{i(12)} y_{j, i(2)}^{2}-\frac{1}{2} \widehat{\beta}_{i(21)} y_{j, i(1)}^{2}
$$

Here

$$
\widehat{\beta}_{i(12)}=\frac{\sum_{j=1}^{M} y_{j, i(1)} y_{j, i(2)}}{\sum_{j=1}^{M} y_{j, i(2)}^{2}} \quad \text { and } \quad \widehat{\beta}_{i(21)}=\frac{\sum_{j=1}^{M} y_{j, i(1)} y_{j, i(2)}}{\sum_{j=1}^{M} y_{j, i(1)}^{2}} .
$$

Importantly the theory for correlation is entirely symmetric in the ways it deals with the two asset returns. In particular the scaling $\left(\sum_{j=1}^{M} y_{j, i(1)}^{2} \sum_{j=1}^{M} y_{j, i(2)}^{2}\right)^{-1}$ adjusts the denominator to make it invariant as we scale either of the asset returns within each time period. This suggests it should be less sensitive to changes in the level of volatility in either of the assets.

Figure 4 shows the realised correlation error

$$
\frac{\sum_{j=1}^{M} y_{j, i(1)} y_{j, i(2)}}{\sqrt{\sum_{j=1}^{M} y_{j, i(1)}^{2} \sum_{j=1}^{M} y_{j, i(2)}^{2}}}-\frac{\int_{\hbar(i-1)}^{\hbar i} \Sigma_{21}(u) \mathrm{d} u}{\sqrt{\int_{\hbar(i-1)}^{\hbar i} \Sigma_{11}(u) \mathrm{d} u \int_{\hbar(i-1)}^{\hbar i} \Sigma_{22}(u) \mathrm{d} u}},
$$

plotted against $n$, together with $95 \%$ confidence intervals generated using the above theory. What becomes very clear is that the precision of the estimators does not vary very much. This conclusion is reinforced as $M$ increases. This means the errors for realised correlations are approximately unconditionally normal.

The bottom plots in Figure 4 give QQ plots for the simulations of the realised correlation based on the asymptotic theory. These plots are based on 2,000 observations. The asymptotic theory is a poor guide for small values of $M$, but for moderate to large values of $M$ it is reasonably accurate.

One possible way of improving the finite sample behaviour of the asymptotic distribution of $\widehat{\rho}_{i(12)}$ is by using the Fisher (1921)-z transformation (e.g. see the exposition in Anderson (1984, 

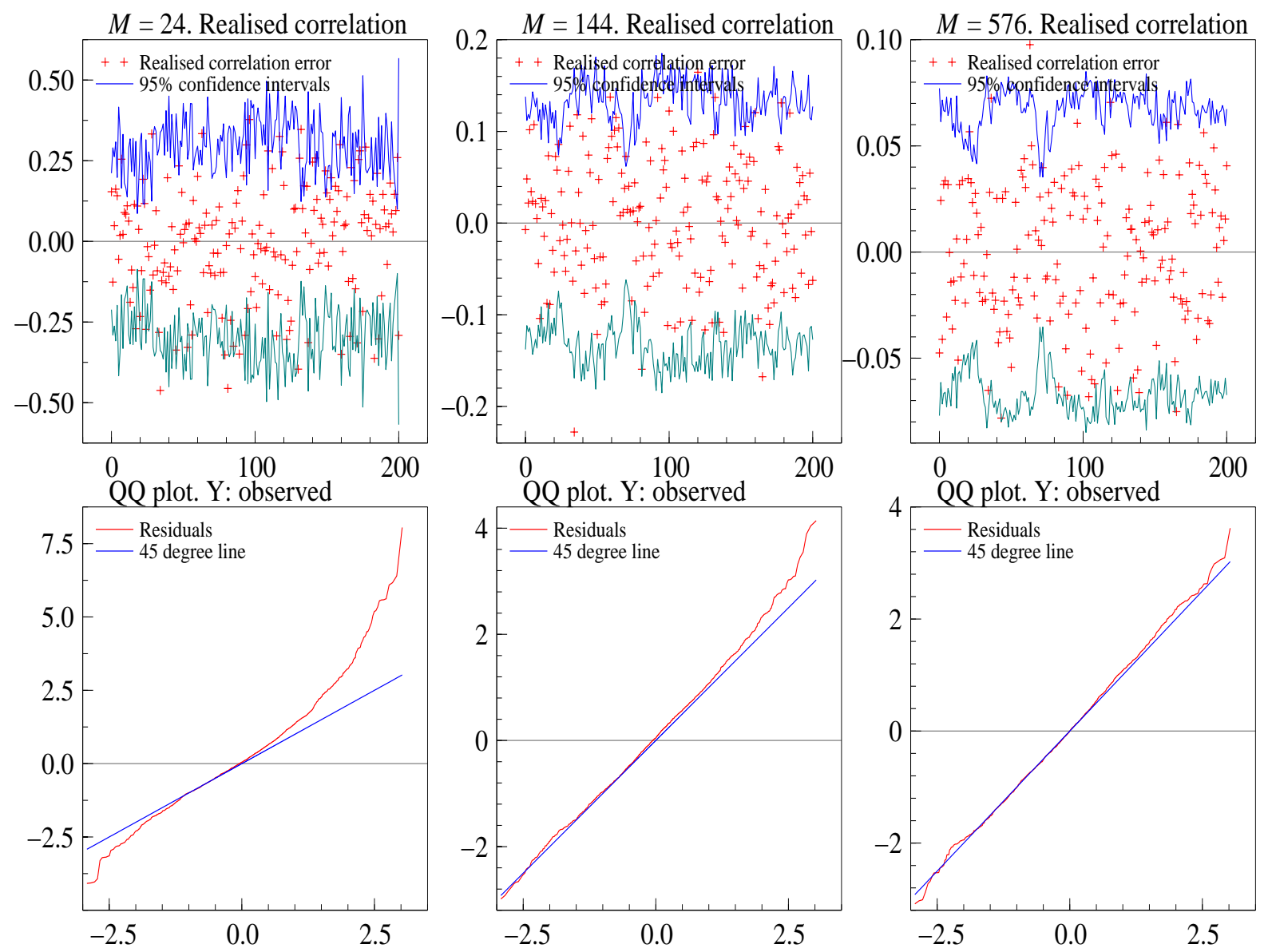

Figure 4: Simulation from a bivariate factor $S V$ model. Top line: drawn is the realised correlation errors of asset one on asset two together with the associated asymptotic standard errors. Bottom line is the corresponding $Q Q$ plot to assess the accuracy of the asymptotic theory. Code is available at: sim_mult.ox

$122-124))$

$$
z_{i(12)}=\frac{1}{2} \log \frac{1+\widehat{\rho}_{i(12)}}{1-\widehat{\rho}_{i(12)}} \quad \text { and } \quad \zeta_{i(12)}=\frac{1}{2} \log \frac{1+\rho_{i(12)}}{1-\rho_{i(12)}} .
$$

Recall Fisher's analysis was based on $M$ multivariate, independent and identically distributed Gaussian data, in which case his transformation has the important feature that $\sqrt{M}\left(z_{i(12)}-\zeta_{i(12)}\right)$ has a standard normal limit distribution and it is well known its asymptotic distribution provides an excellent approximation to the exact distribution (e.g. David (1938)). In our more general case

$$
\frac{z_{i(12)}-\zeta_{i(12)}}{\sqrt{\left\{1-\left(\widehat{\rho}_{i(12)}\right)^{2}\right\}^{-2}\left(\sum_{j=1}^{M} y_{j, i(1)}^{2} \sum_{j=1}^{M} y_{j, i(2)}^{2}\right)^{-1}\left(\sum_{j=1}^{M} x_{j, i}^{2}-\sum_{j=1}^{M-1} x_{j, i} x_{j+1, i}\right)}} \stackrel{L}{\rightarrow} N(0,1) .
$$

Figure 4 repeats the previous experiment but now using the Fisher transformation. This 



Figure 5: Simulation from a bivariate factor SV model. Top line: drawn is the realised correlation error of assets one and two together with the associated asymptotic standard errors and the constant interval based on an incorrect application of Fisher's limit theory. Bottom line is the corresponding $Q Q$ plot to assess the accuracy of the correct asymptotic theory and the Fisher approximation. Code is available at: sim_mult.ox

pictures demonstrate that the Fisher transformation improves the performance of the asymptotic approximation and that the confidence intervals are again made even more stable through time. This is particularly apparent when we look at the cases where $M$ is very high.

Figure 4 also graphs the corresponding confidence intervals generated by Fisher's original theory, which is clearly not asymptotically valid here. The result is a flat confidence interval, generated by $\pm 2 / \sqrt{M}$. This interval is very close to the asymptotically valid ones and actually results in a better behaved QQ plot. This result arises as the realised correlation is not effected by changes in the level of the volatilities in either series, except where they occur within a day. However, in this simulation experiment this does not happen. Consequently the Fisher theory approximately applies. Of course over more sustained periods or with processes with even more dramatic moves in the correlation or volatility structure within days the incorrect application 
of the Fisher theory can be highly misleading.

\subsection{Effectiveness of the theory under leverage effects}

This section will repeat the experiments reported in the previous subsections but this time based on the Feller or Cox, Ingersoll, and Ross (1985) square root process ${ }^{10}$ for the volatility dynamics. This has two aims, to demonstrate that the results we indicated above are not sensitive to the type of volatility processes used in building the model and to explore the effect of leverage terms on the performance of our theory.

We write the dynamics as

$$
\mathrm{d} s_{j}^{*}(t)=\sigma_{j}(t) \mathrm{d} w_{j}(t), \quad j=1, \ldots, m,
$$

but now with

$$
\mathrm{d} \sigma_{j}^{2}(t)=-\lambda_{j}\left\{\sigma_{j}^{2}(t)-\xi_{j}\right\} \mathrm{d} t+\omega_{j} \sigma_{j}(t) \mathrm{d} b_{j}\left(\lambda_{j} t\right), \quad \xi_{j} \geq \omega_{j}^{2} / 2
$$

where $b_{j}(t)$ is a standard Brownian motion process (independent over $j$ ) with

$$
\operatorname{Cor}\left\{b_{j}\left(\lambda_{j} t\right), w_{j}(t)\right\}=\rho_{j} t \sqrt{\lambda_{j}}
$$

The correlation parameters $\rho_{1}, \rho_{2}, \ldots, \rho_{m}$ represent the leverage effect in the model and would be expected to be negative (e.g. Black (1976) and Nelson (1991)). The square root process has a marginal distribution

$$
\sigma_{j}^{2}(t) \sim \Gamma\left(2 \omega_{j}^{-2} \xi_{j}, 2 \omega_{j}^{-2}\right)=\Gamma\left(\nu_{j}, a_{j}\right), \quad \nu_{j} \geq 1
$$

with a mean of $\nu_{j} / a_{j}$ and a variance of $\nu_{j} / a_{j}^{2}$. Throughout this section we again take $\hbar=1$.

Then we repeat our choices for the parameters (76) and (77), which implies

$$
\left(\begin{array}{l}
\omega_{1} \\
\omega_{2} \\
\omega_{3}
\end{array}\right)=\left(\begin{array}{l}
0.63 \\
1.41 \\
1.41
\end{array}\right) \quad \text { and } \quad\left(\begin{array}{l}
\xi_{1} \\
\xi_{2} \\
\xi_{3}
\end{array}\right)=\left(\begin{array}{l}
0.4 \\
1 \\
1.5
\end{array}\right) .
$$

In these experiments we will focus on the $M=576$ case and see how varying between two possible regimes

$$
\left(\begin{array}{l}
\rho_{1} \\
\rho_{2} \\
\rho_{3}
\end{array}\right)=\left(\begin{array}{l}
0 \\
0 \\
0
\end{array}\right), \quad\left(\begin{array}{l}
\rho_{1} \\
\rho_{2} \\
\rho_{3}
\end{array}\right)=\left(\begin{array}{l}
-0.98 \\
-0.98 \\
-0.98
\end{array}\right),
$$

will effect the performance of our asymptotics. The above cases represent the no leverage case and the strong leverage, respectively.

\footnotetext{
${ }^{10}$ In the context of SV models this is often called the Heston (1993) model.
} 

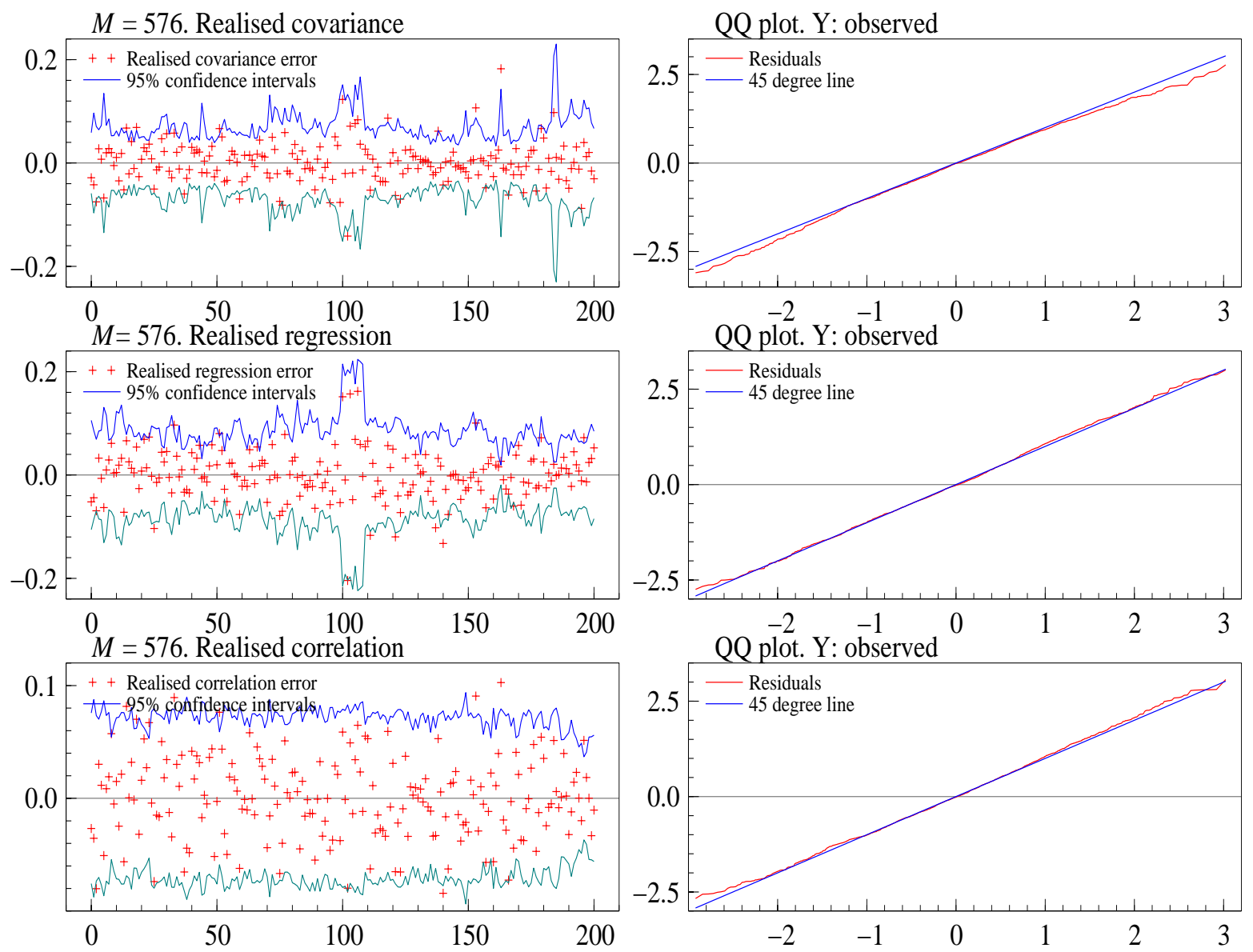

Figure 6: Simulation from a bivariate factor $S V$ model based on the square root volatility processes with no leverage. All calculations are based on $M=576$. Top line: drawn is the realised covariance error with the associated asymptotic standard errors and $Q Q$ plot. Middle line: corresponding results for realised regression. Bottom line: results for realised correlation. Code is available at: sim_mult.ox.

The results in the no leverage case are given in Figure 6 . They show that moving to the diffusion based volatility model does not really change any of the conclusions from the previous subsection — the asymptotics still provide a useful guide to the finite sample behaviour of these statistics. Although not surprising, since this case is covered by our theory, this is a reassuring result.

The results with strong leverage are given in Figure $7^{11}$. They are very much in line with those we reported for the non-leverage case and suggest that our analysis may be robust to this effect. Proving this conjecture turns out to be challenging mathematically even in the univariate case and is the subject of on-going research. In a stimulating piece of work Meddahi (2002) has shown that the effect of leverage on the unconditional mean square error of the

\footnotetext{
${ }^{11}$ In the simulation, common random numbers are used in the leverage and non-leverage cases.
} 

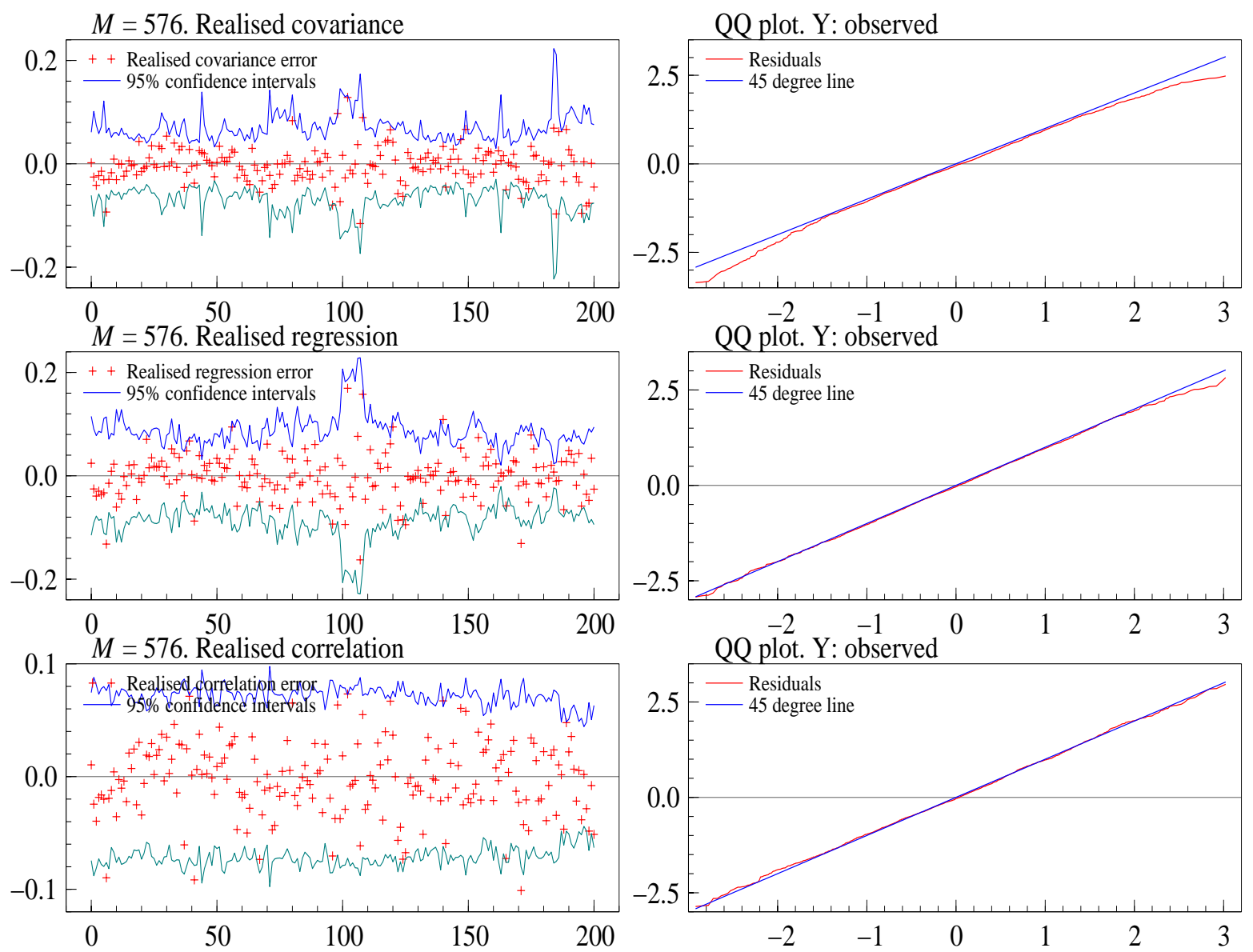

Figure 7: Simulation from a bivariate factor $S V$ model based on the square root volatility processes with high amounts of leverage. All calculations are based on $M=576$. Top line: drawn is the realised covariance error with the associated asymptotic standard errors and $Q Q$ plot. Middle line: corresponding results for realised regression. Bottom line: results for realised correlation. Code is available at: sim_mult.ox

realised covariation error is asymptotically negligible in a wide class of diffusion based volatility models. This again points to the more general result of our asymptotics working in the leverage case.

\section{Empirical illustration}

\subsection{Confidence intervals for realised quantities}

To illustrate some of the empirical features of realised covariation we have used a subset of the return data employed by Andersen, Bollerslev, Diebold, and Labys (2001b), although the Appendix will describe the slightly different adjustments we have made to deal with some missing data. This bivariate series records the United States Dollar/ German Deutsche Mark and Dollar/ Japanese Yen series. It covers the ten year period from 1st December 1986 until 30th November 
1996. The subset we have selected to illustrate our theory starts on February 4th 1991 and covers the next 100 trading days. The original dataset records every 5 minutes the most recent quote to appear on the Reuters screen. It has been kindly supplied to us by Olsen and Associates in Zurich. Throughout the analysis presented in this section we will set $M=144$, thus basing our analysis on 10 minute return data with $\hbar$ representing one day.

(a): realised covariance for DM \& Yen

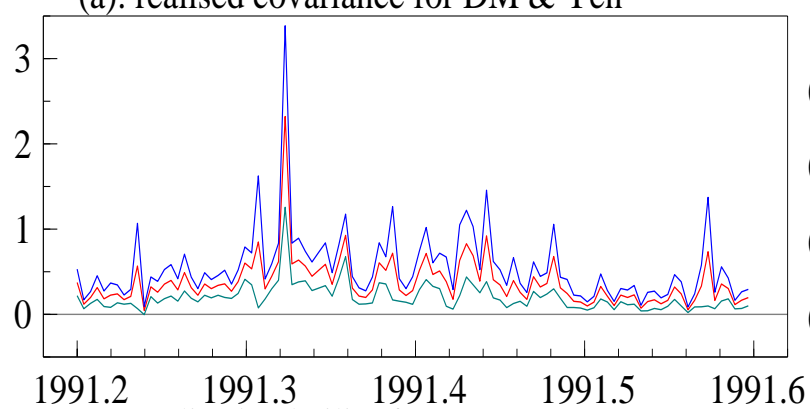

(c): realised volatility for DM
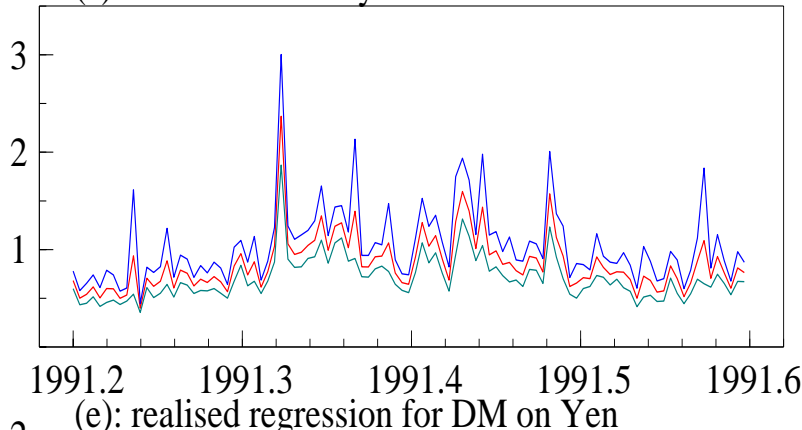

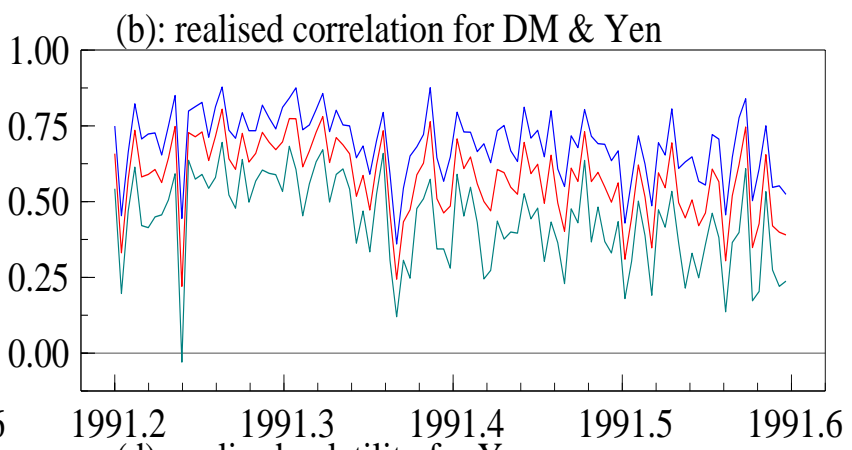

(d): realised volatility for Yen

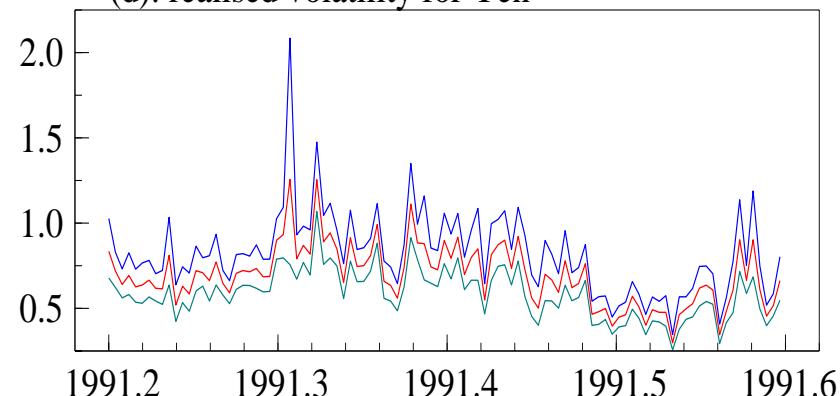

(f): realised regression for Yen on DM

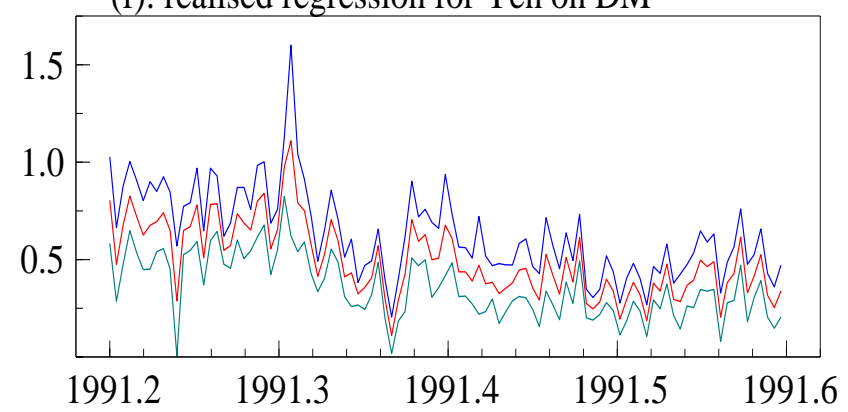

Figure 8: Realised quantities, and their 95\% confidence intervals, for ten minute changes bivariate exchange rate data on the DM and Yen against the Dollar. Data is 4th February 1991 onwards for 100 active trading days. Top left: realised covariance for the DM and Yen. Top right: realised correlation for the DM and Yen. Middle left: realised variance for the DM. Middle right: realised variance for the Yen. Bottom left: regression coefficient for DM on Yen. Bottom right: regression coefficient for Yen on DM. Code: asy_mult_appli.ox.

In the top left graph in Figure 8 we have drawn the realised covariance

$$
\sum_{j=1}^{M} y_{j, i(1)} y_{j, i(2)},
$$

together with the associated $95 \%$ confidence intervals constructed using our asymptotic the- 
ory. These terms move rather violently through this period, while the corresponding realised correlations

$$
\frac{\sum_{j=1}^{M} y_{j, i(1)} y_{j, i(2)}}{\sqrt{\sum_{j=1}^{M} y_{j, i(1)}^{2} \sum_{j=1}^{M} y_{j, i(2)}^{2}}},
$$

given in the top right piece of Figure 8 is actually quite stable. The correlations are not particularly precisely estimated, with the confidence intervals typically being around 0.3 wide (they are computed using (83)). The instability in the realised covariance is caused by movements in the realised volatilities $\sqrt{\sum_{j=1}^{M} y_{j, i(1)}^{2}}$ and $\sqrt{\sum_{j=1}^{M} y_{j, i(2)}^{2}}$, which are given in the middle elements of the Figure. Interestingly the largest shifts in the correlation structure during this time period do not appear at times of particularly large volatility spikes. The bottom graphs also pick up the large movements in the volatility for they show the regressions of the DM on the Yen and the Yen on the DM

$$
\frac{\sum_{j=1}^{M} y_{j, i(1)} y_{j, i(2)}}{\sqrt{\sum_{j=1}^{M} y_{j, i(2)}^{2}}} \text { and } \frac{\sum_{j=1}^{M} y_{j, i(1)} y_{j, i(2)}}{\sqrt{\sum_{j=1}^{M} y_{j, i(1)}^{2}}},
$$

respectively. These move very significantly over time, but again this is mostly due to volatility shifts, not changes in the pattern of correlation.

\subsection{Is the correlation constant through time?}

It is clear that the volatilities of the DM and Yen against the Dollar change very significantly through time and this effects the realised covariances and realised regressions. The integrated correlations, on the other hand, are more stable and an interesting question is whether we can regard them as being constant. Are the variations we see in the time series plot possibly explained by noise? Here we assess this hypothesis formally, employing another feature of our asymptotics - that the asymptotic analysis can be carried out with varying choices of $\hbar$.

To assess the stability of the correlation amongst the exchange rates we compute the daily Fisher transform of the realised correlation

$$
z_{i(12)}=\frac{1}{2} \log \frac{1+\widehat{\rho}_{i(12)}}{1-\widehat{\rho}_{i(12)}} \quad \text { where } \quad \widehat{\rho}_{i(12)}=\frac{\sum_{j=1}^{M} y_{j, i(1)} y_{j, i(2)}}{\sqrt{\sum_{j=1}^{M} y_{j, i(1)}^{2} \sum_{j=1}^{M} y_{j, i(2)}^{2}}}
$$

amongst the rates over the 200 active days again following 4th February 1991. This is then compared with the transformed realised correlation computed once spanning the same 200 days (we could have computed it over the whole sample, but this would risk rejecting the null due to slowly evolving, long term changes in the correlations). To make this more precise we write $n=200$ to denote the total number of days in the sample and then compute

$$
z_{(12)}=\frac{1}{2} \log \frac{1+\widehat{\rho}_{(12)}}{1-\widehat{\rho}_{(12)}} \quad \text { where } \quad \widehat{\rho}_{(12)}=\frac{\sum_{i=1}^{n} \sum_{j=1}^{M} y_{j, i(1)} y_{j, i(2)}}{\sqrt{\left(\sum_{i=1}^{n} \sum_{j=1}^{M} y_{j, i(1)}^{2}\right)\left(\sum_{i=1}^{n} \sum_{j=1}^{M} y_{j, i(2)}^{2}\right)}} .
$$


Ignoring the effect of a single day being overlapping between these two computations in order to simplify the exposition ${ }^{12}$, then as $M \rightarrow \infty$

$$
\frac{\left(z_{i(12)}-z_{(12)}\right)-\left(\zeta_{i(12)}-\zeta_{(12)}\right)}{\sqrt{s_{i}^{2}+s^{2}}} \stackrel{L}{\rightarrow} N(0,1),
$$

where

$$
\begin{gathered}
\zeta_{i(12)}=\frac{1}{2} \log \frac{1+\rho_{i(12)}}{1-\rho_{i(12)}}, \quad \text { and } \quad \rho_{i(12)}=\frac{\int_{\hbar(i-1)}^{\hbar i} \Sigma_{12}(u) \mathrm{d} u}{\sqrt{\int_{\hbar(i-1)}^{\hbar i} \Sigma_{11}(u) \mathrm{d} u} \sqrt{\int_{\hbar(i-1)}^{\hbar i} \Sigma_{22}(u) \mathrm{d} u}}, \\
\zeta_{(12)}=\frac{1}{2} \log \frac{1+\rho_{(12)}}{1-\rho_{(12)}}, \quad \text { and } \quad \rho_{(12)}=\frac{\int_{0}^{\hbar n} \Sigma_{12}(u) \mathrm{d} u}{\sqrt{\int_{0}^{\hbar n} \Sigma_{11}(u) \mathrm{d} u} \sqrt{\int_{0}^{\hbar n} \Sigma_{22}(u) \mathrm{d} u}} .
\end{gathered}
$$

Further $s_{i}^{2}$ is computed as the denominator in (83), while

$$
\begin{aligned}
s^{2}= & \left\{1-\left(\widehat{\rho}_{(12)}\right)^{2}\right\}^{-2}\left\{\left(\sum_{i=1}^{n} \sum_{j=1}^{M} y_{j, i(1)}^{2}\right)\left(\sum_{i=1}^{n} \sum_{j=1}^{M} y_{j, i(2)}^{2}\right)\right\}^{-1} \\
& \times\left\{\left(\sum_{i=1}^{n} \sum_{j=1}^{M} x_{j, i}^{2}\right)-\left(\sum_{i=1}^{n} \sum_{j=1}^{M-1} x_{j, i} x_{j+1, i}\right)\right\},
\end{aligned}
$$

where

$$
x_{j, i}=y_{j, i(1)} y_{j, i(2)}-\frac{1}{2} \widehat{\beta}_{(12)} y_{j, i(2)}^{2}-\frac{1}{2} \widehat{\beta}_{(21)} y_{j, i(1)}^{2},
$$

that is the regressions are based on the whole sample. Under a null hypothesis that the correlation pattern has not changed through time, then

$$
\frac{z_{i(12)}-z_{(12)}}{\sqrt{s_{i}^{2}+s^{2}}} \stackrel{L}{\rightarrow} H_{0} N(0,1) .
$$

We plot $z_{i(12)}-z_{(12)}$ together with twice $\sqrt{s_{i}^{2}+s^{2}}$ in Figure 9. This shows endemic rejection of the null hypothesis, with the correlations moving in discernible patterns throughout the sample. A hypothesis of constant correlations is not maintainable for this dataset.

\section{Conclusions}

In this paper we have developed a distribution theory for realised covariation, a quantity which appears both in finance theory and in a great deal of empirical financial econometrics. Our new theory can be used to derive a feasible limit theory for realised regression and realised correlation. This is robust for it does not need the empirical researcher to specify a model for

\footnotetext{
${ }^{12}$ We could formally ignore the dependence by simply dropping the $i$-th day's data from $\widehat{\rho}_{(12)}$. This would make the statistics asymptotically (as $M \rightarrow \infty$, but with $n$ fixed) independent. This happens without any assumption that the returns between days are independent.
} 


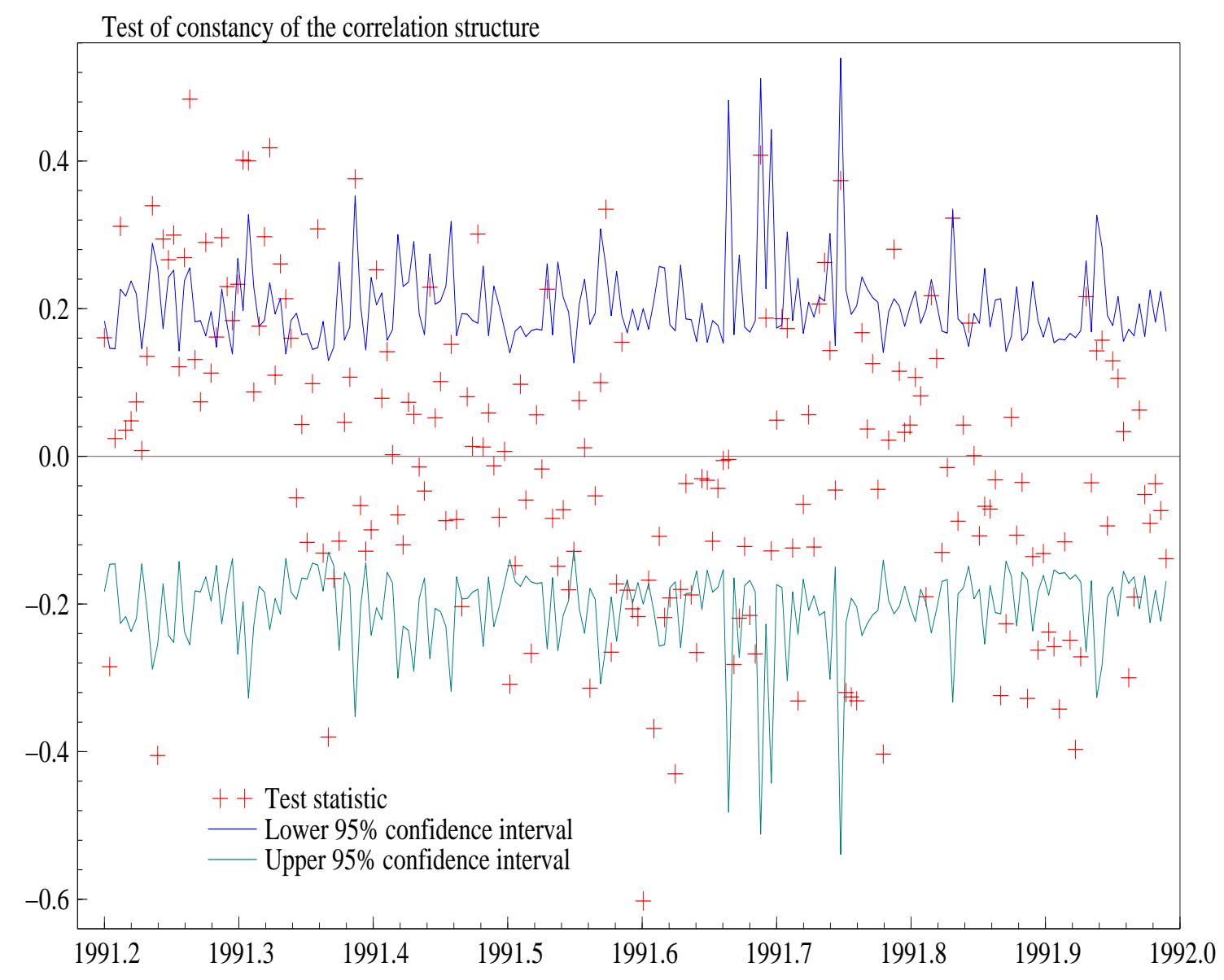

Figure 9: Fisher transformations of the realised correlations. Crosses denote $z_{i(12)}-z_{(12)}$. Comparison is with the daily correlations with the same quantity computed over 200 days. 95\% confidence intervals are based on the mixed Gaussian $M \rightarrow \infty$ limit theory. Code: asy_mult_appli.ox.

the spot covariation or the drift process. In that sense it is semi-parametric. Unlike most of the semi-parametric literature, there are no tuning parameters to choose in our approach, the theory is both easy to code and self-contained. Monte Carlo results suggest the theory may well be useful in practice for it seems a good guide to the finite sample behaviour. Further, the Monte Carlo results conjecture that the main draw back of the theory, the inability of our theory to deal with leverage effects, may not be very important in practice.

Although the paper has made substantial progress, there are many issues which are not resolved. Estimated covariances are used in practice to compute, for example, portfolio selection weights and mean-variance efficiency frontiers. The above theory can be used to calculate a distribution theory for these economically interesting features.

An important theme in theoretical econometrics and statistics is that covariances are not very robust objects, as they are highly sensitive to large movements in asset prices. It maybe 
desirable to construct economic theory and econometrics on more robust quantities such as mean absolute errors. In some recent work Barndorff-Nielsen and Shephard (2001c) have studied the univariate version of this problem, in particular establishing a limiting distribution theory for $M^{-1 / 2} \sum_{j=1}^{M}\left|y_{j, i}\right|$, which is somewhat robust to jumps in the price process. We are currently trying to construct multivariate versions of this type of result.

\section{Acknowledgments}

Ole E. Barndorff-Nielsen's work is supported by CAF (www.caf.dk), which is funded by the Danish Social Science Research Council, and by MaPhySto (www.maphysto.dk), which is funded by the Danish National Research Foundation. Neil Shephard's research is supported by the UK's ESRC through the grant "Econometrics of trade-by-trade price dynamics," which is coded R00023839. All the calculations made in this paper are based on software written by the authors using the Ox language of Doornik (2001). We thank Torben Andersen, Tim Bollerslev, Frank Gerhard and Nour Meddahi for very helpful conversations on this topic.

\section{A Proofs}

\section{A.1 Notation \& semimartingales}

We consider $q$ stochastic processes $y_{\nu}^{*}$, determined by a set of stochastic differential equations

$$
\mathrm{d} y_{\nu}^{*}(t)=\mathrm{d} \alpha_{\nu}^{*}(t)+\gamma_{\nu}^{a}(t) \mathrm{d} w_{a}(t)
$$

with initial condition $y_{\nu}^{*}(0)=0(\nu=1, \ldots, q)$ and $a=1, \ldots, m$ (recall $m$ could be bigger, equal or smaller than $q$ ). Throughout we are using the Einstein summation convention ${ }^{13}$ to indices $a, b, c, d$ but not to the indices $k, l, k^{\prime}, l^{\prime}$. In what is to follow we will write $\gamma_{k l}^{a b}=\gamma_{k}^{a} \gamma_{l}^{b}$, with similar notation for other index combinations. Using this notation the spot covolatility matrix of the SV model is

$$
\Sigma_{k l}(t)=\gamma_{k l}^{a a}(t)
$$

Throughout this section we notice that covering the $i=1$ case is sufficient, for the results for all other values of $i$ follow immediately. In order to simplify the notation we drop reference to $n$ and write $\hbar=t$. Let $M$ denote a positive integer and, for an arbitrary $t \in \mathbf{R}_{+}$, set

$$
\delta=t / M
$$

the time period of the high frequency return.

\footnotetext{
${ }^{13}$ Recall the Einstein summation convention means that if an index is repeated in a single expression then summation over that index is understood.
} 
For any $q$-dimensional semimartingale $X$ with components $X_{\nu}, \nu=1, \ldots, q$, we define higher order variations

$$
\left[X_{\nu_{1}}, \ldots, X_{\nu_{d}}\right](t)=\underset{\delta \downarrow}{\lim }\left[X_{\delta \nu_{1}}, \ldots, X_{\delta \nu_{d}}\right](t),
$$

(provided the limit exists). Here $d$ denotes a positive integer and $\nu_{1}, \ldots, \nu_{d}$ is any set of $d$ indices, each index arbitrarily chosen from $\{1, \ldots, q\}$. Furthermore, $X_{\nu \delta}$ denotes the discrete approximation to $X_{\nu}$ given by

$$
X_{\nu \delta}(s)=X_{\nu}((j-1) \delta) \text { for }(j-1) \delta \leq s<j \delta
$$

and

$$
\left[X_{\delta \nu_{1}}, \ldots, X_{\delta \nu_{d}}\right](t)=\delta^{-d / 2+1} \sum_{j=1}^{M}\left\{X_{\nu_{1}}(\delta j)-X_{\nu_{1}}((j-1) \delta)\right\} \cdots\left\{X_{\nu_{d}}(j \delta)-X_{\nu_{d}}((j-1) \delta)\right\} .
$$

Note that for $q=2$ our notation coincides with the usual notation for the covariation of two semimartingales and we have $\left[X_{\nu \delta}\right]=\left[X_{\nu \delta}, X_{\delta \nu}\right]$ and $\left[X_{\delta}\right]=\left[X_{\delta}, X_{\delta}\right]$ where $X_{\delta}=\left(X_{1 \delta}, \ldots, X_{q \delta}\right)$.

In studying the higher order variations $\left[X_{\delta \nu_{1}}, \ldots, X_{\delta \nu_{d}}\right]$ it is helpful to use the fact, which follows from the multidimensional version of Ito's formula (cf. for instance Protter (1990, p. 74), that for any continuous semimartingales $Y_{t}^{1}, \ldots, Y_{t}^{m}$ (with starting value 0 ) we have

$$
Y_{t}^{1} \cdots Y_{t}^{m}=\sum_{j=1}^{m} \int_{0}^{t} \prod_{k \neq j} Y_{s}^{k} \mathrm{~d} Y_{s}^{j}+\sum_{1 \leq j<k \leq m} \int_{0}^{t} \prod_{l \neq j, k} Y_{s}^{l} \mathrm{~d}\left[Y^{j}, Y^{k}\right]_{s} .
$$

For $m=2$ this reduces to

$$
Y_{t}^{1} Y_{t}^{2}=\int_{0}^{t} Y_{s}^{1} \mathrm{~d} Y_{s}^{2}+\int_{0}^{t} Y_{s}^{2} \mathrm{~d} Y_{s}^{1}+\int_{0}^{t} \mathrm{~d}\left[Y^{1}, Y^{2}\right]_{s}
$$

while for $m=4$

$$
\begin{aligned}
Y_{t}^{1} Y_{t}^{2} Y_{t}^{3} Y_{t}^{4}= & \int_{0}^{t} Y_{s}^{1} Y_{t}^{2} Y_{t}^{3} \mathrm{~d} Y^{4}+\int_{0}^{t} Y_{s}^{1} Y_{t}^{2} Y_{t}^{4} \mathrm{~d} Y^{3}+\int_{0}^{t} Y_{s}^{1} Y_{t}^{3} Y_{t}^{4} \mathrm{~d} Y^{2}+\int_{0}^{t} Y_{t}^{2} Y_{t}^{3} Y^{4} \mathrm{~d} Y_{s}^{1} \\
& +\int_{0}^{t} Y_{s}^{1} Y_{t}^{2} \mathrm{~d}\left[Y^{3}, Y^{4}\right]_{s}+\int_{0}^{t} Y_{s}^{1} Y_{t}^{3} \mathrm{~d}\left[Y^{2}, Y^{4}\right]_{s}+\int_{0}^{t} Y_{s}^{1} Y_{t}^{4} \mathrm{~d}\left[Y^{2}, Y^{3}\right]_{s} \\
& +\int_{0}^{t} Y_{s}^{2} Y_{t}^{3} \mathrm{~d}\left[Y^{1}, Y^{4}\right]_{s}+\int_{0}^{t} Y_{s}^{2} Y_{t}^{3} \mathrm{~d}\left[Y^{1}, Y^{3}\right]_{s}+\int_{0}^{t} Y_{s}^{3} Y_{t}^{4} \mathrm{~d}\left[Y^{1}, Y^{2}\right]_{s} .
\end{aligned}
$$

\section{A.2 Proofs of theorems 1 and 2}

\section{Proof of Theorem 1 and 2.}

We are interested in the limiting behaviour of

$$
\left[y_{k \delta}^{*}, y_{l \delta}^{*}\right](t)=\sum_{j=1}^{M} y_{k j} y_{l j}=\sum_{j=1}^{M}\left\{y_{k}^{*}(\delta j)-y_{k}^{*}((j-1) \delta)\right\}\left\{y_{l}^{*}(j \delta)-y_{l}^{*}((j-1) \delta)\right\},
$$


when the processes $\alpha_{k}^{*}, \gamma_{k}^{a}$ are considered given by conditioning.

To the asymptotic order considered the limit behaviour of $\left[y_{\delta}^{*}\right]$ is dominated by the infinitesimal variation of the Brownian motion $w$, so that - as we shall show - the variation of the (vector) process $\alpha^{*}$ does not influence the limit laws.

Suppose initially that $\alpha^{*}$ is identically 0 , in which case

$$
y_{k j}=\int_{(j-1) \delta}^{j \delta} \gamma_{k}^{a}(s) \mathrm{d} w_{a}(s)
$$

The summands in (92) are independent and since the processes $\gamma_{k}^{a}$ are locally of bounded variation the matrix $\left[y_{\delta}^{*}\right]$ must asymptotically be normally distributed. (Detailed verification of this follows standard reasoning and is therefore omitted here.) The task is thus to determine the asymptotic mean and variance of $\left[y_{\delta}^{*}\right]$.

Let

$$
\Gamma_{k l}(t)=\int_{0}^{t} \gamma_{k l}^{a a}(s) \mathrm{d} s
$$

and

$$
\Gamma_{k l j}=\int_{(j-1) \delta}^{j \delta} \gamma_{k l}^{a a}(s) \mathrm{d} s .
$$

By (93) and (89) we find $\mathrm{E}\left\{y_{k j} y_{l j}\right\}=\Gamma_{k l j}$ and hence we have

$$
\mathrm{E}\left\{\left[y_{k \delta}^{*}, y_{l \delta}^{*}\right](t)\right\}=\Gamma_{k l}(t)=\Sigma_{k l}(t) .
$$

Furthermore, for any indices $k, l, k^{\prime}, l^{\prime}$ in $\{1, \ldots, q\}$,

$$
\begin{aligned}
\operatorname{Cov}\left\{\left[y_{k \delta}^{*}, y_{l \delta}^{*}\right](t),\left[y_{k^{\prime} \delta}^{*}, y_{l^{\prime} \delta}^{*}\right](t)\right\} & =\mathrm{E}\left\{\sum_{j^{\prime}=1}^{M}\left(y_{k j^{\prime}} y_{l j^{\prime}}-\Gamma_{k l j^{\prime}}\right) \sum_{j=1}^{M}\left(y_{k^{\prime} j} y_{l^{\prime} j}-\Gamma_{k^{\prime} l^{\prime} j}\right)\right\} \\
& =\sum_{j=1}^{M} \mathrm{E}\left\{y_{k j} y_{l j} y_{k^{\prime} j} y_{l^{\prime} j}\right\}-\sum_{j=1}^{M} \Gamma_{k l j} \Gamma_{k^{\prime} l^{\prime} j} .
\end{aligned}
$$

Consider now the case $j=1$. Using (91) and similarly for other index combinations, we find

$$
\begin{aligned}
\mathrm{E}\left\{y_{k 1} y_{l 1} y_{k^{\prime} 1} y_{l^{\prime} 1}\right\} & =\int_{0}^{\delta} \mathrm{E}\left\{\int_{0}^{u} \int_{0}^{u} \gamma_{k}^{a}(s) \gamma_{l}^{b}(s) \mathrm{d} b_{a}(s) \mathrm{d} b_{b}(s)\right\} \gamma_{k^{\prime}}^{c}(u) \gamma_{l^{\prime}}^{c}(u) \mathrm{d} u \quad[6] \\
& =\int_{0}^{\delta} \gamma_{k^{\prime} l^{\prime}}^{c c}(u) \int_{0}^{u} \gamma_{k l}^{a a}(s) \mathrm{d} s \mathrm{~d} u
\end{aligned}
$$

the symbol [6] indicating that $\mathrm{E}\left\{y_{k j} y_{l j} y_{k^{\prime} j} y_{l^{\prime} j}\right\}$ equals the sum of the term given plus 5 similar terms obtained via permutation of the indices $k, l, k^{\prime}, l^{\prime}$. Continuing the calculation we have

$$
\begin{aligned}
\mathrm{E}\left\{y_{k 1} y_{l 1} y_{k^{\prime} 1} y_{l^{\prime} 1}\right\} & =\int_{0}^{\delta} \gamma_{k^{\prime} l^{\prime}}^{c c}(u) \Gamma_{k l}(u) \mathrm{d} u \\
& =\int_{0}^{\delta} \gamma_{k^{\prime} l^{\prime}}^{c c}(u) \Gamma_{k l}(u) \mathrm{d} u+\int_{0}^{\delta} \gamma_{k l}^{c c}(u) \Gamma_{k^{\prime} l^{\prime}}(u) \mathrm{d} u
\end{aligned}
$$




$$
\begin{aligned}
& +\int_{0}^{\delta} \gamma_{l l^{\prime}}^{c c}(u) \Gamma_{k k^{\prime}}(u) \mathrm{d} u+\int_{0}^{\delta} \gamma_{k k^{\prime}}^{c c}(u) \Gamma_{l l^{\prime}}(u) \mathrm{d} u \\
& +\int_{0}^{\delta} \gamma_{k^{\prime} l}^{c c}(u) \Gamma_{k l^{\prime}}(u) \mathrm{d} u+\int_{0}^{\delta} \gamma_{k l^{\prime}}^{c c}(u) \Gamma_{k^{\prime} l}(u) \mathrm{d} u
\end{aligned}
$$

Next we note that

$$
\frac{\mathrm{d}}{\mathrm{d} s}\left\{\Gamma_{k l}(s) \Gamma_{k^{\prime} l^{\prime}}(s)\right\}=\gamma_{k l}^{c c}(s) \Gamma_{k^{\prime} l^{\prime}}(s)+\gamma_{k^{\prime} l^{\prime}}^{c c}(s) \Gamma_{k l}(s)
$$

or, in other words,

$$
\int_{0}^{\delta} \gamma_{k^{\prime} l^{\prime}}^{c c}(u) \Gamma_{k l}(u) \mathrm{d} u+\int_{0}^{\delta} \gamma_{k l}^{c c}(u) \Gamma_{k^{\prime} l^{\prime}}(u) \mathrm{d} u=\Gamma_{k l}(\delta) \Gamma_{k^{\prime} l^{\prime}}(\delta)
$$

The terms for other values of the indices behave similarly and all in all we obtain

$$
\operatorname{Cov}\left\{\left[y_{k \delta}^{*}, y_{l \delta}^{*}\right](t),\left[y_{k^{\prime} \delta}^{*}, y_{l^{\prime} \delta}^{*}\right](t)\right\}=\sum_{j=1}^{M}\left(\Gamma_{k k^{\prime} j} \Gamma_{l l^{\prime} j}+\Gamma_{k l^{\prime} j} \Gamma_{l k^{\prime} j}\right)
$$

Now, when $\delta \rightarrow 0$ the sum in (96) turns into an integral and behaves as $\delta \Omega_{k l, k^{\prime} l^{\prime}}(t)$ where

$$
\Omega_{k l, k^{\prime} l^{\prime}}(t)=\int_{0}^{t}\left\{\gamma_{k k^{\prime}}^{a a}(s) \gamma_{l l^{\prime}}^{c c}(s)+\gamma_{k l^{\prime}}^{a a}(s) \gamma_{l k^{\prime}}^{c c}(s)\right\} \mathrm{d} s,
$$

as stated in Theorem 1.

Turning then to Theorem 2, recall from (94) that

$$
\operatorname{Cov}\left\{\left[y_{k \delta}^{*}, y_{l \delta}^{*}\right](t),\left[y_{k^{\prime} \delta}^{*}, y_{l^{\prime} \delta}^{*}\right](t)\right\}=\sum_{j=1}^{M} \mathrm{E}\left\{y_{k j} y_{l j} y_{k^{\prime} j} y_{l^{\prime} j}\right\}-\sum_{j=1}^{M} \Gamma_{k l j} \Gamma_{k^{\prime} l^{\prime} j}
$$

From the previous discussion we have

$$
\delta^{-1} \operatorname{Cov}\left\{\left[y_{k \delta}^{*}, y_{l \delta}^{*}\right](t),\left[y_{k^{\prime} \delta}^{*}, y_{l^{\prime} \delta}^{*}\right](t)\right\} \rightarrow \Omega_{k l, k^{\prime} l^{\prime}}(t)
$$

On the other hand, arguing as above it is seen that

$$
\delta^{-1} \sum_{j=1}^{M} \mathrm{E}\left\{y_{k j} y_{l j} y_{k^{\prime} j} y_{l^{\prime} j}\right\} \rightarrow \int_{0}^{t}\left\{\gamma_{k l}^{a a}(s) \gamma_{k^{\prime} l^{\prime}}^{c c}(s)+\gamma_{k k^{\prime}}^{a a}(s) \gamma_{l l^{\prime}}^{c c}(s)+\gamma_{k l^{\prime}}^{a a}(s) \gamma_{l k^{\prime}}^{c c}(s)\right\} \mathrm{d} s
$$

and

$$
\delta^{-1} \sum_{j=1}^{M} \Gamma_{k l j} \Gamma_{k^{\prime} l^{\prime} j} \rightarrow \int_{0}^{t} \gamma_{k l}^{a a}(s) \gamma_{k^{\prime} l^{\prime}}^{c c}(s) \mathrm{d} s
$$

and, moreover, that

$$
\left[y_{\delta k}^{*}, y_{\delta l}^{*}, y_{\delta k^{\prime}}^{*}, y_{\delta l^{\prime}}^{*}\right]=\delta^{-1} \sum_{j=1}^{M} y_{k j} y_{l j} y_{k^{\prime} j} y_{l^{\prime} j},
$$

must converge in probability to the same limit as $\delta^{-1} \sum_{j=1}^{M} \mathrm{E}\left\{y_{k j} y_{l_{j}} y_{k^{\prime} j} y_{l^{\prime} j}\right\}$. Thus to obtain a consistent estimator of $\Omega_{k l, k^{\prime} l^{\prime}}(t)$ it suffices to find a consistent estimator of $\int_{0}^{t} \gamma_{k l}^{a a}(s) \gamma_{k^{\prime} l^{\prime}}^{c c}(s) \mathrm{d} s$. The quantity

$$
\sum_{j=1}^{M-1} y_{k j} y_{l j} y_{k^{\prime}(j+1)} y_{l^{\prime}(j+1)}
$$


solves this problem, as is seen by again applying formula (91).

To prove that the same limiting laws hold when the mean processes $\alpha_{\nu}^{*}$ are not 0 but satisfy condition (A) we first note that

$$
y_{k j} y_{l j}=\alpha_{k j} \alpha_{l j}+\alpha_{k j} y_{0 l j}+\alpha_{l j} y_{0 k j}+y_{0 k j} y_{0 l j}
$$

where

$$
y_{0 k j}=\int_{(j-1) \delta}^{j \delta} \gamma_{k}^{a}(s) \mathrm{d} b_{a}(s) .
$$

Under condition (A), $\alpha_{k j}$ is $o(\sqrt{\delta})$ uniformly in $k$ and $j$. Furthermore we have

$$
\alpha_{k j} y_{0 l j}+\alpha_{l j} y_{0 k j} \sim N\left(0, \alpha_{k j}^{2} \Gamma_{l l j}+\alpha_{l j}^{2} \Gamma_{k k j}\right) .
$$

Consequently,

$$
\begin{aligned}
{\left[y_{k \delta}^{*}, y_{l \delta}^{*}\right](t) } & =\sum_{j=1}^{M} \alpha_{k j} \alpha_{l j}+\sum_{j=1}^{M}\left(\alpha_{k j} y_{0 l j}+\alpha_{l j} y_{0 k j}\right)+\sum_{j=1}^{M} y_{0 k j} y_{0 l j} \\
& =o(\sqrt{\delta})+o_{p}(\sqrt{\delta})+\left[y_{0 k \delta}^{*}, y_{0 l \delta}^{*}\right](t)
\end{aligned}
$$

where

$$
\left[y_{0 k \delta}^{*}, y_{0 l \delta}^{*}\right](t)=\sum_{j=1}^{M} y_{0 k j} y_{0 l j} .
$$

It follows that, conditionally, $\delta^{-1 / 2}\left\{\left[y_{\delta}^{*}, y_{\delta}^{*}\right]-\Gamma\right\}$ has the same limit law as $\delta^{-1 / 2}\left\{\left[y_{0 \delta}^{*}, y_{0 \delta}^{*}\right]-\Gamma\right\}$ and the latter is as given in Theorem 1 .

An analogous argument applies to Theorem 2.

\section{B Data manipulation}

The Olsen group have kindly made available to us a dataset which records every five minutes the most recent quote to appear on the Reuters screen from 1st December 1986 until 30th November 1996. When prices are missing they have interpolated them. Details of this processing are given in Dacorogna, Gencay, Muller, Olsen, and Pictet (2001). The same dataset was analysed by Andersen, Bollerslev, Diebold, and Labys (2001b). We follow the extensive work of Torben Andersen and Tim Bollerslev on this dataset, who remove much of the times when the market is basically closed. This includes almost all of the weekend, while they have taken out most US holidays. The result is what we will regard as a single time series of length 705,313 observations. Although many of the breaks in the series have been removed, sometimes there are sequences of very small price changes caused by, for example, unmodelled non-US holidays or data feed breakdowns. We deal with this by adding a Brownian bridge simulation to sequences of data 

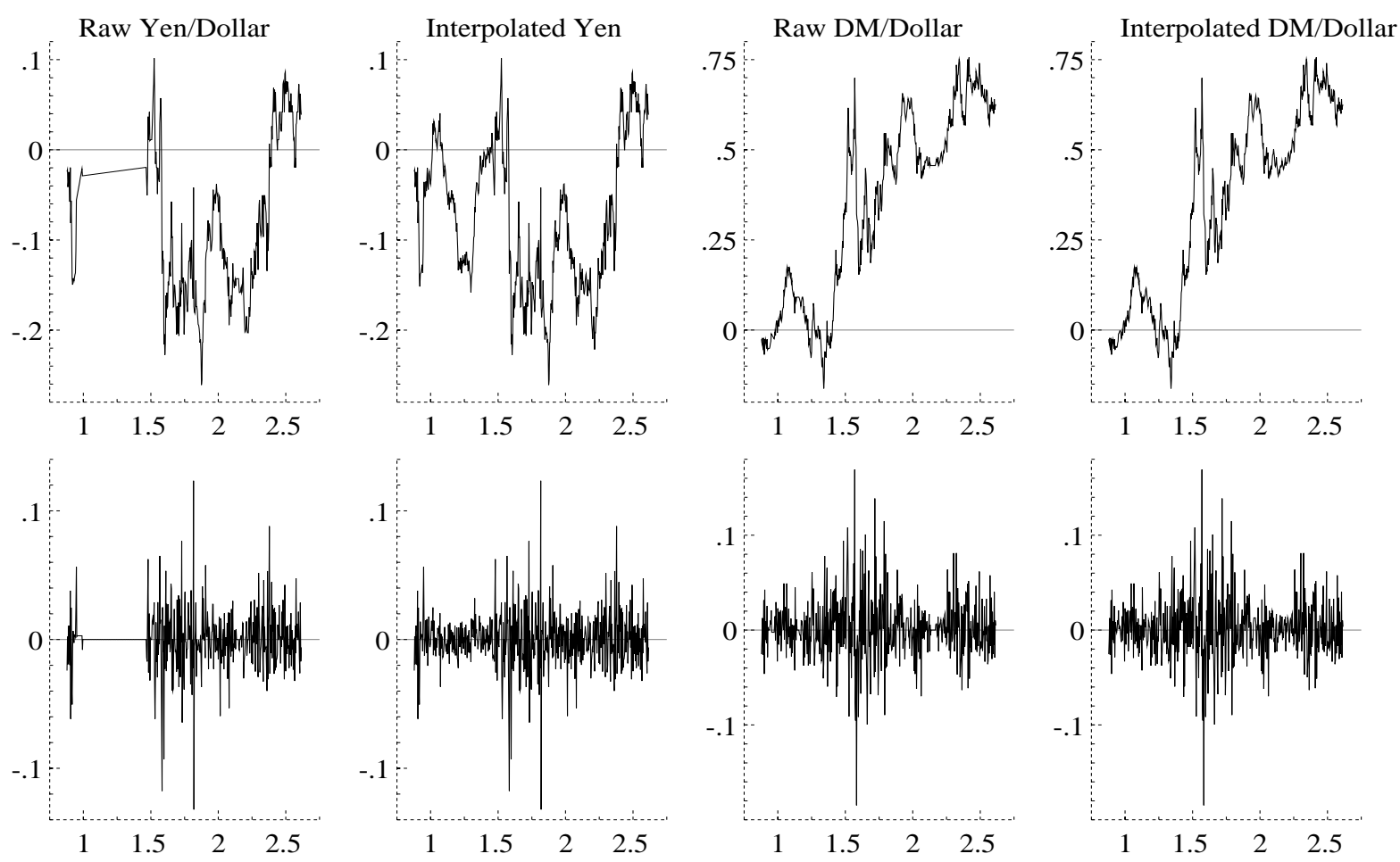

Figure 10: Top line of graphs are the raw and interpolated data using a Brownian bridge interpolator. Bottom line of graphs is the corresponding returns. The x-axes are marked off in days.

where at each time point the absolute change in a five minute period is below $0.01 \%$. That is, when this happens, we interpolate prices stochastically, adding a Brownian bridge with a standard deviation of 0.01 for each time period. By using a bridge process we are not affecting the long run trajectory of prices, while the impact on realised volatility is very small indeed. We have used this stochastic method here in order to be consistent with our other work on this topic where this effect is important. It is illustrated in Figure 10, which shows the first 500 observations in the Dollar/DM series we have used in this paper and another series which is for the Yen/Dollar. Later stretches of the data have fewer breaks in them, but this graph illustrates the effects of our intervention. Clearly our approach is ad hoc. However, a proper statistical modelling of these breaks is very complicated due to their many causes and the fact that our dataset is enormous.

\section{References}

Andersen, T. G. and T. Bollerslev (1998). Answering the skeptics: yes, standard volatility models do provide accurate forecasts. International Economic Review 39, 885-905. 
Andersen, T. G., T. Bollerslev, and F. X. Diebold (2002). Parametric and nonparametric measurement of volatility. In Y. Ait-Sahalia and L. P. Hansen (Eds.), Handbook of Financial Econometrics. Amsterdam: North Holland. Forthcoming.

Andersen, T. G., T. Bollerslev, F. X. Diebold, and H. Ebens (2001). The distribution of realised stock return volatility. Journal of Financial Economics 61, 43-76.

Andersen, T. G., T. Bollerslev, F. X. Diebold, and P. Labys (2001b). The distribution of exchange rate volatility. Journal of the American Statistical Association 96, 42-55.

Andersen, T. G., T. Bollerslev, F. X. Diebold, and P. Labys (2001a). Modeling and forecasting realized volatility. Unpublished paper: Department of Economics, Duke University.

Anderson, T. W. (1984). An Introduction to Multivariate Statistical Analysis (2 ed.). New York: John Wiley and Sons.

Andreou, E. and E. Ghysels (2001). Rolling-sampling volatility estimators: some new theoretical, simulation and empirical results. Journal of Business and Economic Statistics 19. Forthcoming.

Back, K. (1991). Asset pricing for general processes. Journal of Mathematical Economics 20, 371-395.

Bai, X., J. R. Russell, and G. C. Tiao (2000). Beyond Merton's utopia: effects of non-normality and dependence on the precision of variance estimates using high-frequency financial data. Unpublished paper: Graduate School of Business, University of Chicago.

Barndorff-Nielsen, O. E. and N. Shephard (2001b). How accurate is the asymptotic approximation to the distribution of realised volatility? Unpublished paper, Nuffield College, Oxford.

Barndorff-Nielsen, O. E. and N. Shephard (2001a). Non-Gaussian Ornstein-Uhlenbeck-based models and some of their uses in financial economics (with discussion). Journal of the Royal Statistical Society, Series B 63, 167-241.

Barndorff-Nielsen, O. E. and N. Shephard (2001c). Realised power variation and stochastic volatility. Unpublished paper: Nuffield College, Oxford.

Barndorff-Nielsen, O. E. and N. Shephard (2002a). Econometric analysis of realised volatility and its use in estimating stochastic volatility models. Journal of the Royal Statistical Society, Series B 64. Forthcoming.

Barndorff-Nielsen, O. E. and N. Shephard (2002b). Estimating quadratic variation using realised variance. Journal of Applied Econometrics. Forthcoming. 
Black, F. (1976). Studies of stock price volatility changes. Proceedings of the Business and Economic Statistics Section, American Statistical Association, 177-181.

Campbell, J. Y., A. W. Lo, and A. C. MacKinlay (1997). The Econometrics of Financial Markets. Princeton, New Jersey: Princeton University Press.

Chib, S., F. Nardari, and N. Shephard (1999). Analysis of high dimensional multivariate stochastic volatility models. Unpublished paper: Nuffield College, Oxford.

Chriss, N. and W. Morokoff (1999). Volatility and variance swaps. Risk 12, 55-59.

Cochrane, J. H. (2001). Asset Pricing. Princeton: Princeton University Press.

Comte, F. and E. Renault (1998). Long memory in continuous-time stochastic volatility models. Mathematical Finance 8, 291-323.

Cox, J. C., J. E. Ingersoll, and S. A. Ross (1985). A theory of the term structure of interest rates. Econometrica 53, 385-407.

Dacorogna, M. M., R. Gencay, U. A. Muller, R. B. Olsen, and O. V. Pictet (2001). An Introduction to High-Frequency Finance. San Diego: Academic Press.

David, F. N. (1938). Tables of the Ordinates and Probability Integral of the Distribution of the Correlation Coefficient in Small Samples. Cambridge: Cambridge University Press.

Demeterfi, K., E. Derman, M. Kamal, and J. Zou (1999). A guide to volatility and variance swaps. Journal of Derivatives 6, 9-32.

Diebold, F. X. and M. Nerlove (1989). The dynamics of exchange rate volatility: a multivariate latent factor ARCH model. Journal of Applied Econometrics 4, 1-21.

Doornik, J. A. (2001). Ox: Object Oriented Matrix Programming, 3.0. London: Timberlake Consultants Press.

Duffie, D., J. Pan, and K. Singleton (2000). Transform analysis and asset pricing for affine jump-diffusions. Econometrica 68, 1343-1376.

Fisher, R. A. (1921). On the probable error of a coefficient of correlation deduced from a small sample. Metron 1, 3-32.

Florens-Zmirou, D. (1993). On estimating the diffusion coefficient from discrete observations. Journal of Applied Probability 30, 790-804.

Foster, D. P. and D. B. Nelson (1996). Continuous record asymptotics for rolling sample variance estimators. Econometrica 64, 139-174. 
Genon-Catalot, V., C. Laredo, and D. Picard (1992). Non-parametric estimation of the diffusion coefficient by wavelet methods. Scandinavian Journal of Statistics 19, 317-335.

Ghysels, E., A. C. Harvey, and E. Renault (1996). Stochastic volatility. In C. R. Rao and G. S. Maddala (Eds.), Statistical Methods in Finance, pp. 119-191. Amsterdam: North-Holland.

Hansen, B. E. (1995). Regression with non-stationary volatility. Econometrica 63, 1113-1132.

Heston, S. L. (1993). A closed-form solution for options with stochastic volatility, with applications to bond and currency options. Review of Financial Studies 6, 327-343.

Howison, S. D., A. Rafailidis, and H. O. Rasmussen (2000). A note on the pricing and hedging of volatility derivatives. Unpublished paper: Mathematical Institute, University of Oxford.

Hubalek, F. and E. Nicolato (2001). On multivariate extensions of Lévy driven OrnsteinUhlenbeck type stochastic volatility models and multi-asset options. Working Paper, University of Aarhus.

Jacod, J. and A. N. Shiryaev (1987). Limit Theorems for Stochastic Processes. Springer-Verlag: Berlin.

Lintner, J. (1965). The valuation of risk assets and the selection of risky investments in stock portfolios and capital budgets. Review of Economics and Statistics 47, 13-37.

Liu, Q. (2002). Estimating betas from high-frequency data. Unpublished paper: Kellogg Graduate School of Management, Northwestern University.

Lutkepohl, H. (1996). Handbook of Matrices. Chichester: Wiley.

Meddahi, N. (2002). A theoretical comparison between integrated and realized volatilities. Journal of Applied Econometrics. Forthcoming.

Meddahi, N. and E. Renault (1996). Aggregation and marginalization of GARCH and stochastic volatility models. Unpublished paper: CREST-INSEE.

Meddahi, N. and E. Renault (2002). Temporal aggregation of volatility models. Journal of Econometrics. Forthcoming.

Nelson, D. B. (1991). Conditional heteroskedasticity in asset pricing: a new approach. Econometrica 59, 347-370.

Newey, W. K. and K. D. West (1987). A simple positive semi-definite, heteroskedasticity and autocorrelation consistent covariance matrix. Econometrica 55, 703-708.

Pagan, A. R. and A. Ullah (1999). Nonparametric Econometrics. Cambridge: Cambridge University Press. 
Pitt, M. K. and N. Shephard (1999). Time varying covariances: a factor stochastic volatility approach (with discussion). In J. M. Bernardo, J. O. Berger, A. P. Dawid, and A. F. M. Smith (Eds.), Bayesian Statistics 6, pp. 547-570. Oxford: Oxford University Press.

Protter, P. (1990). Stochastic Integration and Differential Equations: A New Approach. New York: Springer-Verlag.

Shephard, N. (1996). Statistical aspects of ARCH and stochastic volatility. In D. R. Cox, D. V. Hinkley, and O. E. Barndorff-Nielsen (Eds.), Time Series Models in Econometrics, Finance and Other Fields, pp. 1-67. London: Chapman \& Hall. 